DIVISION OF THE HUMANITIES AND SOCIAL SCIENCES

CALIFORNIA INSTITUTE OF TECHNOLOGY

PASADENA, CALIFORNIA 91125

ARBITRAGE-BASED PRICING WHEN

VOLATILITY IS STOCHASTIC

Peter Bossaerts

California Institute of Technology

Eric Ghysels

Université de Montréal and CIRANO, Montréal

Christian Gouriéroux

CREST, Paris

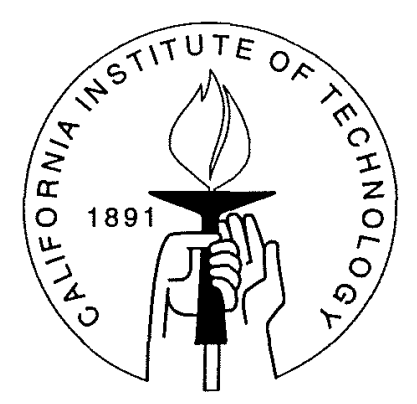

SOCIAL SCIENCE WORKING PAPER 977

July 1996 


\title{
Arbitrage-Based Pricing When Volatility Is Stochastic
}

\author{
Peter Bossaerts Eric Ghysels Christian Gouriéroux
}

\begin{abstract}
In one of the early attempts to model stochastic volatility, Clark [1973] conjectured that the size of asset price movements is tied to the rate at which transactions occur. To formally analyze the econometric implications, he distinguished between transaction time and calendar time. The present paper exploits Clark's strategy for a different purpose, namely, asset pricing. It studies arbitrage-based pricing in economies where: (i) trade takes place in transaction time, (ii) there is a single state variable whose transactiontime price path is binomial, (iii) there are riskfree bonds with calendar-time maturities, and (iv) the relation between transaction time and calendar time is stochastic. The state variable could be interpreted in various ways. E.g., it could be the price of a share of stock, as in Black and Scholes [1973], or a factor that summarizes changes in the investment opportunity set, as in Cox, Ingersoll and Ross [1985] or one that drives changes in the term structure of interest rates (Ho and Lee [1986], Heath, Jarrow and Morton [1992]). Property (iv) generally introduces stochastic volatility in the process of the state variable when recorded in calendar time. The paper investigates the pricing of derivative securities with calendar-time maturities. The restrictions obtained in Merton [1973] using simple buy-and-hold arbitrage portfolio arguments do not necessarily obtain. Conditions are derived for all derivatives to be priced by dynamic arbitrage, i.e., for market completeness in the sense of Harrison and Pliska [1981]. A particular class of stationary economies where markets are indeed complete is characterized.
\end{abstract}

JEL Classification : D52, G13.

Keywords: Incomplete Markets, Transaction Time, Change of Time, Stochastic Volatility. 


\title{
Arbitrage-Based Pricing When Volatility Is Stochastic*
}

\author{
Peter Bossaerts $^{\dagger} \quad$ Eric Ghysels ${ }^{\ddagger} \quad$ Christian Gouriéroux $^{\S}$
}

\section{Introduction}

One of the early examples of stochastic volatility models is Clark [1973]. He suggested that asset price movements should be tied to the rate at which transactions occur. To obtain the formal econometric implications of this conjecture, he distinguished explicitly between two clocks, one measuring calendar time, and another measuring transaction time. Clark's strategy has hitherto been relatively unexploited to study derivative securities pricing. This paper is meant to fill the gap.

We study the arbitrage pricing restrictions in economies where trade takes place according to a (discrete) transactions clock which differs from the standard calendar clock. In transaction time, calendar-time ticks are stochastic. Riskfree bonds with calendar-time maturities are traded. There is a single state variable whose process in transaction time is binomial. We are interested in obtaining unique prices for derivatives using arbitrage arguments. In other words, we are investigating conditions for markets to be complete in the sense of Harrison and Pliska [1981].

We assume that calendar-time ticks coincide with (randomly chosen) transaction-time ticks. (The term "tick" is used here in the common sense of the discrete movement of the hands of a clock.) Most of the paper focuses on trade in transaction time. The assumption that calendar-time ticks can occur only upon a transaction-time tick, however, allows us to study also portfolio rebalancing in calendar time and its pricing implications. When portfolio rebalancing is restricted to calendar time and the probability of a calendar-time tick at any point in (transaction) time is bounded away from zero and one, the economies in this paper are horrendously incomplete. The representation of the process of the state variable is that of a tree with an infinite number of branches at every step.

${ }^{*}$ The paper benefited from comments in the UCLA Theory Workshop and during the Workshop on the Mathematics of Finance in Montreal (April 30-May 3 1996). Hélyette Geman's comments made us extend the scope of the theory beyond stock option pricing.

${ }^{\dagger}$ California Institute of Technology

łUniversité de Montréal and CIRANO (Montréal)

$\S_{\text {CREST (Paris). }}$ 
The state variable could be interpreted in many ways. For instance, it could be the price of a share of stock, as in Black and Scholes [1973]. It could also be a factor that summarizes changes in the investment opportunity set, as in Cox, Ingersoll and Ross [1985], or one that determines the term structure of interest rates, as in Ho and Lee [1986] and Heath, Jarrow and Morton [1992].

Because of the random nature of calendar-time ticks, the state variable will generally exhibit stochastic volatility when recorded in calendar time. Hence, we effectively study arbitrage pricing under stochastic volatility. In such an environment, it is generally claimed that, in the absence of a security with a price that is perfectly correlated with volatility, dynamic arbitrage arguments are insufficient to price derivatives. By considering stochastic volatility as emerging from the randomness of calendar-time ticks on the transaction clock, we provide a different view on the issue of market incompleteness.

Empirically, there appears to be high correlation between the transaction count and stochastic volatility. See, e.g., Harris [1986], [1987] and Tauchen and Pitts [1983], or, more recently, Ghysels and Jasiak [1995]. In fact, the appearance of the two processes is sufficiently similar for some to model the duration between two transactions by borrowing succesful approaches (in particular, GARCH) from the volatility literature. See Engle and Russell [1996]. This paper is theoretical. As in Clark [1973], we take the extreme view that stochastic volatility is entirely generated by the random relationship between transaction and calendar time and we study the pricing implications of such a view.

We could assume that the (implicit) riskfree rate in transaction time is strictly positive (we will also, however, investigate the case where the riskfree rate is zero). This assumption makes it costly to hold on to a (static) arbitrage position in the face of transactions, and, hence, volatility. The cost may be interpreted, for instance, as the effect of margin calls. The most profound implication of this assumption is to invalidate many of the option pricing restrictions derived in Merton [1973]. European put-call parity, for instance, fails to obtain, confirming empirical violations (see, e.g., Kamara and Miller [1995]).

We investigate necessary conditions for derivatives written on the state variable to be priced by dynamic arbitrage. In other words, we study whether and when markets could be complete. We prove that interest rates (calendar-time bond yields) have to be stochastic for arbitrage arguments to generate unique derivatives prices. Interest rates not only have to be stochastic in transaction time; they must not be constant when recorded in calendar time. We provide a counterexample that stochastic interest rates do not constitute a sufficient condition for arbitrage pricing.

Hull and White [1987] derived a pricing formula for stock options under constant interest rates, using a risk-neutral probability measure for which the disturbance process of the stock price and that of the volatility are independent. Without restricting our attention to pricing under a single risk-neutral probability measure, we study the effects of analogous assumptions in our context. We assume that prices of traded assets allow for a state price process such that (i) the (implicit) transaction-time interest rate is constant, 
and (ii) the state variable (which could be interpreted as the stock price) and the calendartime tick processes are independent under the corresponding risk-neutral probability measure. We demonstrate that this makes the economy generically incomplete. As a by-product, we show what assumptions on the bond price processes are sufficient for there to be a risk-neutral measure under which the state variable and calendar-time tick processes are independent, and, hence, for Hull and White's pricing technique to make sense.

The class of economies where it is possible to price derivatives by arbitrage is not empty. We characterize a subclass, where state price processes are stationary. In it, derivatives prices solve a complex difference equation. We also demonstrate how to imply the (unique) risk-neutral probabilities (which are really normalized Arrow-Debreu securities prices) from a set of bond price processes.

One could wonder why we take the transaction-time clock as given, instead of deriving it as the equilibrium to an economy that is modeled at some deeper level. The reason is simple: we are not interested in equilibrium price and transaction processes per se. We study the restrictions on price processes that are imposed in the presence of arbitrageurs who wish to exploit perceived arbitrage opporunities by (potentially) rebalancing a hedge portfolio. Since arbitrageurs cannot rebalance but in transaction time, our taking transaction time as the base clock seems only natural. This also clarifies why we shall not allow there to be more than one calendar-time tick per transaction-time period. Finer calendar-time measurement would be irrelevant for an arbitrageur: she could not possibly use them to rebalance hedge portfolios.

Since a hedge portfolio consists almost by definition of more than one security (in the present case, bonds with different calendar-time maturities), our transaction time is essentially defined as the count of occasions when it is possible to simultaneously trade in each of the component securities. The fact that such a count is possible at all is not a trivial requirement. If the state variable is the price of a share of stock, for instance, whose process is binomial when recorded in its own transaction time (the count of the stock's transactions), then the only realistic way for a hedge portfolio consisting of bonds to become rebalanceable in the stock's transaction time would be for the bonds to be traded continuously. ${ }^{1}$

Therefore, our notion of transaction time is essentially the count of occasions such that: (i) it is feasible to trade in bonds with calendar-time maturities, (ii) the state variable's values lie on a binomial tree. The main contribution of the present paper, then, is to point out that new hedging opportunities are created when trading takes place according to the nonstandard clock that this count generates.

Time deformation has been used before to facilitate computation of prices of derivatives, but the implications of the possibility to trade according to a different clock have

\footnotetext{
${ }^{1}$ In most countries, however, riskfree bonds (in the form of government securities or other money market securities), are indeed traded much more frequently than stock.
} 
not yet been investigated. For a comprehensive example of the use of time deformation in the calculation of prices, see Geman and Yor [1993]. Their paper actually mentions the idea of trading according to different clocks ("business time scale"; see p. 351), but does not exploit its implications for option pricing. ${ }^{2}$

The remainder of the paper is organized as follows. Section 2 introduces the economy. Section 3 presents the basic issues. Section 4 studies simple arbitrage restrictions on standard options (puts and calls). Section 5 provides necessary conditions for derivatives to be priceable by arbitrage. In Section 6, an example is given that these conditions are not sufficient. Section 7 studies the case where transaction-time interest rates are constant and the state variable and calendar-time tick processes are independent under a risk-neutral probability measure. Section 8 characterizes a class of economies where derivatives can be priced by arbitrage. Section 9 concludes with a list of open questions.

\section{The Economy}

First some definitions. Transaction time is denoted by $t=0,1,2, \ldots$ Uncertainty in the economy is generated by two binomial processes, $X_{t}$ and $Z_{t}$, both taking values in $\{0,1\}$.

1. $\left\{X_{t}\right\}_{t \geq 0}$ is referred to as the calendar-time tick process. Calendar time is defined as:

$$
\xi_{t}=\sum_{\tau=0}^{t} X_{\tau}
$$

2. $\left\{Z_{t}\right\}_{t \geq 0}$ is referred to as the state variable jump process; it drives the evolution of the "state variable" (to be discussed shortly).

Securities prices will be measurable in the information filtration generated by $\left\{X_{t}\right.$, $\left.Z_{t}\right\}_{t \geq 0}$. Let $\mathcal{F}_{t}$ denote the information set at time $t$. Let $P$ denote the probability measure associated with the probability space on which $X_{t}$ and $Z_{t}$ live and let $P_{t-1}$ denote the probability measure conditional on $\mathcal{F}_{t-1}$. We assume: $0<P_{t-1}\left\{X_{t}=1\right\}<1$, $0<P_{t-1}\left\{Z_{t}=1\right\}<1$. In Section 8 , though, we shall examine the consequence of cases where the conditional probability of the event $\left\{X_{t}=1\right\}$ equals 1 .

We introduce a state variable, whose value at $t$ is denoted $s_{t}$, and whose evolution is derived from that of $Z_{t}$, as follows. There are positive constants $u$ and $d(u>d)$ such that:

$$
s_{t}= \begin{cases}s_{t-1} u & \text { if } Z_{t}=1, \\ s_{t-1} d & \text { if } Z_{t}=0 .\end{cases}
$$

(We could make $u$ and $d$ time-dependent, or even path-dependent, but the added complexity does not introduce new economic insights.) Notice that the logarithm of the state

\footnotetext{
${ }^{2}$ Geman and Yor used time-changed Bessel processes to compute path-dependent option price formulas. This approach has been further explored in Geman and Yor [1995], Leblanc and Scaillet [1995] and Delbaen and Shirakawa [1996].
} 
variable process $\left(\ln s_{t}-\ln s_{t-1}\right)$ is conditionally homoscedastic if $P_{t-1}\left\{Z_{t}=1\right\}$ is constant over time.

It is interesting to examine the behavior of the state variable in calendar time, i.e., across increments in the process $\xi_{t}$. Let:

$$
\begin{aligned}
& \tilde{u}=\ln u, \\
& \tilde{d}=\ln d .
\end{aligned}
$$

D.efine:

$$
t(\xi)=\min \left\{t: \xi_{t}=\xi\right\} .
$$

The calendar-time state variable process $s_{\xi}^{*}(\xi=0,1,2, \ldots)$ is determined as follows:

$$
s_{\xi}^{*}=s_{t(\xi)} \text {. }
$$

We have:

$$
\ln s_{\xi}^{*}=\ln s_{\xi-1}^{*}+U_{\xi}^{*},
$$

where

$$
U_{\xi}^{*}=\sum_{\tau=t(\xi-1)}^{t(\xi)-1}\left(Z_{\tau+1}(\tilde{u}-\tilde{d})+\tilde{d}\right) .
$$

Now assume the following.

Assumption 2.1: the processes $\left\{X_{t}\right\}_{t \geq 0}$ and $\left\{Z_{t}\right\}_{t \geq 0}$ are mutually independent;

Assumption 2.2: the $Z_{t}$ are independent and identically distributed over time;

Assumption 2.3: $E\left[Z_{t+1}(\tilde{u}-\tilde{d})+\tilde{d} \mid \mathcal{F}_{t}\right]=0$.

We are interested in the conditional variance of $U_{\xi}^{*}$. The assumptions allow us to focus on the conditional second moment. Let $\sigma^{2}$ denote the (time-invariant) conditional variance of $Z_{t+1}(\tilde{u}-\tilde{d})+\tilde{d}$. Let $\mathcal{G}_{\xi-1}$ denote the information generated up to calendar time $\xi-1$. This information set is generated by the sequences $\left\{X_{\tau}\right\}_{\tau=0, \ldots, t(\xi-1)}$ and $\left\{Z_{\tau}\right\}_{\tau=0, \ldots, t(\xi-1)}$, where $t(\xi-1)$ is the minimal time $t$ at which $\xi_{t}=\xi-1$.

Theorem 1 Under Assumptions 2.1-2.3,

$$
E\left[\left(U_{\xi}^{*}\right)^{2} \mid \mathcal{G}_{\xi-1}\right]=\sigma^{2} E\left[t(\xi)-t(\xi-1) \mid \mathcal{G}_{\xi-1}\right] .
$$

Proof: see Appendix.

Notice that (1) typifies a process with stochastic volatility: the conditional variance can be written as the product of a volatility parameter and the conditional expectation of a positive random scaling factor. We therefore have obtained a stochastic volatility 
model through time deformation, as in Clark [1973]. For more explicit analysis of time deformation and its relation with stochastic volatility, see, e.g., Madan and Seneta [1990] and Ghysels and Jasiak [1995].

This translation into calendar time is a good occasion to illustrate how the introduction of a new clock effectively generates a new information filtration. In calendar time, the relevant information filtration is $\left\{\mathcal{G}_{\xi}\right\}_{\xi}$; in transaction time, it is $\left\{\mathcal{F}_{t}\right\}_{t}$. Under the former, markets are incomplete. We will show that markets may be complete under the new filtration. In short, time changes are equivalent to changes in the information filtration; since completeness hinges critically on the information, it should come as no surprise that we can reach different conclusions depending on the notion of time used.

At this point, we must emphasize an important fact: the information sets are not necessarily strictly ordered. It is easy to see how transaction time generates information which is not available in calendar time. But the reverse is also possible. Take an example where there are bonds with calendar-time maturities whose yields are constant in calendar time but stochastic in transaction time. (Section 5 will discuss this case in more detail.) Now consider realizations for which $t(\xi)\left(=\min \left\{t: \xi_{t}=\xi\right\}\right)=t^{*}$, in which case we would compare $\mathcal{G}_{\xi}$ with $\mathcal{F}_{t^{*}}$. Future bond prices, at $\xi+1, \xi+2, \ldots$, are in $\mathcal{G}_{\xi}$, but bond prices at $t^{*}+1, t^{*}+2, \ldots$ are not in $\mathcal{F}_{t^{*}}$.

In fact, if bond prices are known for some $t>t^{*}$, this may be an indication that a calendar-time tick will occur at that point. Hence, strict subsidiarity of $\mathcal{G}_{\xi}$ to $\mathcal{F}_{t^{*}}$ may imply that the arbitrageurs know beforehand the path of calendar-time ticks on the transaction-time clock. We are not assuming that.

Continuing with the specification of our economy, we do not necessarily assume that there is a security that is riskfree in transaction time. Letting $b_{t}$ denote the (often only implicit) price of a one-period pure-discount bond with face value of $\$ 1$ (this price may not be unique), we will impose:

$$
b_{t} \leq 1 .
$$

The case where $b_{t}<1$, all $t$, has profound implications. See Section 4.

We do assume, however, the existence of a set of pure discount bonds with calendartime maturities. At maturity, they pay $\$ 1 . m$ denotes maturity, expressed in calendar time $(m=1,2,3, \ldots) . B_{t}^{m}$ is the time- $t$ price of a bond with $m$ calendar-time ticks till maturity. We add: $m=0$, and set:

$$
B_{t}^{0}=1,
$$

all $t$.

Time- $t$ securities prices are measurable in $\mathcal{F}_{t}$. We wish to make explicit how prices change as a function of $X_{t}$ and $Z_{t}$, in addition to $\mathcal{F}_{t-1}$. Whence the following notation:

$$
B_{t}^{m}=B_{t}^{m}\left(X_{t}, Z_{t}, \mathcal{F}_{t-1}\right) .
$$


Sometimes, the information in $\mathcal{F}_{t-1}$ that is relevant to determine $B_{t}^{m}$ may be limited, e.g., to $X_{t-1}$. We then write:

$$
B_{t}^{m}=B_{t}^{m}\left(X_{t}, Z_{t}, X_{t-1}\right)
$$

We also study stationary economies, in which:

$$
B_{t}^{m}=B^{m}\left(X_{t}, Z_{t}, X_{t-1}\right)
$$

(see Section 8).

Given the low dimensionality of the stochastic processes driving the uncertainty in the economy, bond price processes cannot be set arbitrarily. Absence of arbitrage opportunities imposes restrictions. Applying a well-known result from Harrison and Kreps [1979], we have:

Lemma 2 In the absence of arbitrage opportunities, there exist processes $\left\{q_{t}^{X, Z}\right\}_{t \geq 0}(X=$ $0,1 ; Z=0,1)$, such that, for all $m>0$ :

$$
\begin{aligned}
B_{t}^{m}= & q_{t}^{1,1} B_{t+1}^{m-1}\left(1,1, \mathcal{F}_{t}\right)+q_{t}^{1,0} B_{t+1}^{m-1}\left(1,0, \mathcal{F}_{t}\right) \\
& +q_{t}^{0,1} B_{t+1}^{m}\left(0,1, \mathcal{F}_{t}\right)+q_{t}^{0,0} B_{t+1}^{m}\left(0,0, \mathcal{F}_{t}\right)
\end{aligned}
$$

with $0<q_{t}^{X, Z}<1$, all $t, X, Z$.

(Proof: see Appendix.)

$q_{t}^{X, Z}$ is the time- $t$ price implicit in bond prices of the (Arrow-Debreu) security that pays $\$ 1$ if $X_{t+1}=X$ and $Z_{t+1}=Z$, and $\$ 0$ otherwise. It is also often referred to as the price of the state $X, Z$. It may not be unique. The purpose of this paper is precisely to determine when they are.

In analogy with the notation for bond prices, we shall use:

$$
q_{t}^{X, Z}=q_{t}^{X, Z}\left(X_{t}, Z_{t}, \mathcal{F}_{t-1}\right) .
$$

Sometimes,

$$
q_{t}^{X, Z}=q_{t}^{X, Z}\left(X_{t}, Z_{t}, X_{t-1}\right)
$$

or even:

$$
q_{t}^{X, Z}=q^{X, Z}\left(X_{t}, Z_{t}, X_{t-1}\right)
$$

Using this notation, we can rewrite (2):

$$
\begin{aligned}
& B_{t}^{m}\left(X_{t}, Z_{t}, \mathcal{F}_{t-1}\right) \\
& \quad q_{t}^{1,1}\left(X_{t}, Z_{t}, \mathcal{F}_{t-1}\right) B_{t+1}^{m-1}\left(1,1, \mathcal{F}_{t}\right)+q_{t}^{1,0}\left(X_{t}, Z_{t}, \mathcal{F}_{t-1}\right) B_{t+1}^{m-1}\left(1,0, \mathcal{F}_{t}\right) \\
& \quad+q_{t}^{0,1}\left(X_{t}, Z_{t}, \mathcal{F}_{t-1}\right) B_{t+1}^{m}\left(0,1, \mathcal{F}_{t}\right)+q_{t}^{0,0}\left(X_{t}, Z_{t}, \mathcal{F}_{t-1}\right) B_{t+1}^{m}\left(0,0, \mathcal{F}_{t}\right)
\end{aligned}
$$


The state variable, $s_{t}$, may (but need not) be the price of a traded security, such as a share of stock. If so, Lemma 2 will also restrict its evolution. In other words, $u$ and $d$ will be restricted through:

$$
s_{t}=\left(q_{t}^{1,1}+q_{t}^{0,1}\right) s_{t} u+\left(q_{t}^{1,0}+q_{t}^{0,0}\right) s_{t} d .
$$

To better understand the nature of bond price processes that are consistent with absence of arbitrage (Lemma 2), consider an extreme case, where:

$$
q_{t}^{X, Z}=q^{X, Z}
$$

all $t$. Then, $B_{t}^{m}$ solves the following difference equation:

$$
\begin{aligned}
B_{t}^{m}\left(X_{t}, Z_{t}, \mathcal{F}_{t-1}\right)= & q^{1,1} B_{t+1}^{m-1}\left(1,1, \mathcal{F}_{t}\right)+q^{1,0} B_{t+1}^{m-1}\left(1,0, \mathcal{F}_{t}\right) \\
& +q^{0,1} B_{t+1}^{m}\left(0,1, \mathcal{F}_{t}\right)+q^{0,0} B_{t+1}^{m}\left(0,0, \mathcal{F}_{t}\right) .
\end{aligned}
$$

Applying this to $m=1$ produces:

$$
B_{t}^{1}\left(X_{t}, Z_{t}, \mathcal{F}_{t-1}\right)=q^{1,1}+q^{1,0}+q^{0,1} B_{t+1}^{1}\left(0,1, \mathcal{F}_{t}\right)+q^{0,0} B_{t+1}^{1}\left(0,0, \mathcal{F}_{t}\right)
$$

This difference equation has multiple solutions. Some of them are explosive: $B_{t}^{1} \uparrow \infty$. Such solutions correspond to bubbles. To see this, take the case where $B_{t}^{1}\left(X_{t}, Z_{t}, \mathcal{F}_{t-1}\right)$ $=\tilde{B}_{t}^{1}$, a deterministic function of $t$. Then:

$$
\tilde{B}_{t}^{1}=\left(q^{1,1}+q^{1,0}\right)+\left(q^{0,1}+q^{0,0}\right) \tilde{B}_{t+1}^{1},
$$

where $q^{0,1}+q^{0,0}<1$. This is a forward equation which admits explosive behavior.

The only stationary solution to (5) is:

$$
B_{t}^{1}\left(X_{t}, Z_{t}, \mathcal{F}_{t-1}\right)=B^{1}=\frac{q^{1,1}+q^{1,0}}{1-\left(q^{0,1}+q^{0,0}\right)} .
$$

With this solution $B^{1}$, bond prices of all maturities will also be constant. Take $m=2$. We deduce from (2) that:

$$
B_{t}^{2}\left(X_{t}, Z_{t}, \mathcal{F}_{t-1}\right)=\left(q^{1,1}+q^{1,0}\right) B^{1}+q^{0,1} B_{t+1}^{2}\left(0,1, \mathcal{F}_{t}\right)+q^{0,0} B_{t+1}^{2}\left(0,0, \mathcal{F}_{t}\right) .
$$

This equation also admits a constant solution $B^{2}$ with the property:

$$
B^{2}=\left(B^{1}\right)^{2} .
$$

Iterating over $m$, we obtain:

$$
B^{m}=\left(B^{1}\right)^{m}
$$

While generating constant interest rates in calendar time, this example is not very interesting, because it implies that the evolution of bond prices in transaction time only depend on the residual maturity in calendar time, and not on transaction time. The 
bond price process is binomial, depending only on the calendar-time tick process; the time- $t+1$ payoff on the bond with maturity $m$ (as of time $t$ ) becomes:

$$
\begin{cases}B^{m-1} & \text { if } X_{t+1}=1 \\ B^{m} & \text { if } X_{t+1}=0\end{cases}
$$

In this paper, we shall ignore explosive bond price paths if there exist stationary ones that are compatible with absence of arbitrage. In other words, we do not investigate equilibria with bond price bubbles. As a matter of fact, we thereby make our search for economies with complete markets more difficult. As will be clear from Section 8, it is fairly easy to find examples of stationary economies that are complete conditional on a calendar-time tick. The problem is that such economies are generally incomplete in states of the world where there is no calendar-time tick. These economies would readily become complete, however, if bond prices were allowed to wander in arbitrary ways off their stationary path during spells of transactions in-between two calendar-time ticks.

A final remark about bond prices. Consider the general case in (5) again. If we substitute for $B_{t+1}^{1}\left(0,1, \mathcal{F}_{t}\right)$ and $B_{t+1}^{1}\left(0,0, \mathcal{F}_{t}\right)$, we must be careful. Mechanically, we would replace with the following:

$$
\begin{aligned}
& B_{t+1}^{1}\left(0,1, \mathcal{F}_{t}\right)=q^{1,1}+q^{1,0}+q^{0,1} B_{t+2}^{1}\left(0,1, \mathcal{F}_{t+1}\right)+q^{0,0} B_{t+2}^{1}\left(0,0, \mathcal{F}_{t+1}\right) \\
& B_{t+1}^{1}\left(0,0, \mathcal{F}_{t}\right)=q^{1,1}+q^{1,0}+q^{0,1} B_{t+2}^{1}\left(0,1, \mathcal{F}_{t+1}\right)+q^{0,0} B_{t+2}^{1}\left(0,0, \mathcal{F}_{t+1}\right)
\end{aligned}
$$

Somehow, however, we must make clear that $\mathcal{F}_{t+1}$ in (7) differs from that in (8). In (7),

$$
\mathcal{F}_{t+1}=\mathcal{F}_{t} \wedge\left\{X_{t+1}=0, Z_{t+1}=1\right\}
$$

in $(8)$,

$$
\mathcal{F}_{t+1}=\mathcal{F}_{t} \wedge\left\{X_{t+1}=0, Z_{t+1}=0\right\}
$$

If such ambiguities arise, we shall be explicit. Hence, after one recursion, we would write (5) as follows:

$$
\begin{aligned}
B_{t}^{1}\left(X_{t}\right. & \left., Z_{t}, \mathcal{F}_{t-1}\right) \\
= & \left(q^{1,1}+q^{1,0}\right)+\left(q^{0,1}+q^{0,0}\right)\left(q^{1,1}+q^{1,0}\right) \\
& +q^{0,1}\left(q^{0,1} B_{t+2}^{1}\left(0,1, \mathcal{F}_{t} \wedge\left\{X_{t+1}=0, Z_{t+1}=1\right\}\right)\right. \\
& \left.+q^{0,0} B_{t+2}^{1}\left(0,0, \mathcal{F}_{t} \wedge\left\{X_{t+1}=0, Z_{t+1}=1\right\}\right)\right) \\
& +q^{0,0}\left(q^{0,1} B_{t+2}^{1}\left(0,1, \mathcal{F}_{t} \wedge\left\{X_{t+1}=0, Z_{t+1}=0\right\}\right)\right. \\
& \left.+q^{0,0} B_{t+2}^{1}\left(0,0, \mathcal{F}_{t} \wedge\left\{X_{t+1}=0, Z_{t+1}=0\right\}\right)\right) .
\end{aligned}
$$

\section{Basic Issues}

Can a derivative with calendar-time maturity whose payoff depends on the state variable be priced by a dynamic arbitrage argument based on the riskfree bonds? This is the question we set out to answer. 
Pricing by dynamic arbitrage requires that the one-period payoff on the derivative be spanned by payoffs on a certain number of bonds. The price of the derivative must equal the value of the hedge portfolio for there to be no arbitrage opportunities. The arbitrage price will be unique.

Let $c_{t}^{m}$ denote the time- $t$ price of a derivative with (calendar-time) maturity $m$ (as of time $t$ ) and whose payoff depends on the value of the state variable at maturity. If a calendar-time tick occurs, maturity is reduced from $m$ to $m-1$. We prescribe what $c_{t}^{0}$ is (the value of the derivative when the maturity is reduced at $t$ from 1 to 0 ). E.g., for a call option with exercise price $k$,

$$
c_{t}^{0}=\max \left(0, s_{t}-k\right) .
$$

Assume that $c_{t}^{m}$ is measurable in $\mathcal{F}_{t}$. Analogous with the notation of the previous section, we shall write:

$$
c_{t}^{m}=c_{t}^{m}\left(X_{t}, Z_{t}, \mathcal{F}_{t-1}\right) \text {. }
$$

Using bonds with maturities $m_{1}, m_{2}, m_{3}$ and $m_{4}$ to construct the hedge portfolio, we can introduce the following definition.

Definition: The derivative's price $c_{t}^{m}$ is determined by arbitrage if there exists a solution $\left(w_{t}^{1}, w_{t}^{2}, w_{t}^{3}, w_{t}^{4}\right)$, measurable in $\mathcal{F}_{t}$, to the following system of equations:

$$
\begin{aligned}
c_{t+1}^{m-1}\left(1,1, \mathcal{F}_{t}\right)= & w_{t}^{1} B_{t+1}^{m_{1}-1}\left(1,1, \mathcal{F}_{t}\right)+w_{t}^{2} B_{t+1}^{m_{2}-1}\left(1,1, \mathcal{F}_{t}\right) \\
& +w_{t}^{3} B_{t+1}^{m_{3}-1}\left(1,1, \mathcal{F}_{t}\right)+w_{t}^{4} B_{t+1}^{m_{4}-1}\left(1,1, \mathcal{F}_{t}\right) \\
c_{t+1}^{m-1}\left(1,0, \mathcal{F}_{t}\right)= & w_{t}^{1} B_{t+1}^{m_{1}-1}\left(1,0, \mathcal{F}_{t}\right)+w_{t}^{2} B_{t+1}^{m_{2}-1}\left(1,0, \mathcal{F}_{t}\right) \\
& +w_{t}^{3} B_{t+1}^{m_{3}-1}\left(1,0, \mathcal{F}_{t}\right)+w_{t}^{4} B_{t+1}^{m_{4}-1}\left(1,0, \mathcal{F}_{t}\right) \\
c_{t+1}^{m}\left(0,1, \mathcal{F}_{t}\right)= & w_{t}^{1} B_{t+1}^{m_{1}}\left(0,1, \mathcal{F}_{t}\right)+w_{t}^{2} B_{t+1}^{m_{2}}\left(0,1, \mathcal{F}_{t}\right) \\
& +w_{t}^{3} B_{t+1}^{m_{3}}\left(0,1, \mathcal{F}_{t}\right)+w_{t}^{4} B_{t+1}^{m_{4}}\left(0,1, \mathcal{F}_{t}\right) \\
c_{t+1}^{m}\left(0,0, \mathcal{F}_{t}\right)= & w_{t}^{1} B_{t+1}^{m_{1}}\left(0,0, \mathcal{F}_{t}\right)+w_{t}^{2} B_{t+1}^{m_{2}}\left(0,0, \mathcal{F}_{t}\right) \\
& +w_{t}^{3} B_{t+1}^{m_{3}}\left(0,0, \mathcal{F}_{t}\right)+w_{t}^{4} B_{t+1}^{m_{4}}\left(0,0, \mathcal{F}_{t}\right)
\end{aligned}
$$

These are four equations, each representing one particular state of the world at $t+1$. From top to bottom: $\left(X_{t+1}=1, Z_{t+1}=1\right),\left(X_{t+1}=1, Z_{t+1}=0\right),\left(X_{t+1}=0, Z_{t+1}=1\right)$, $\left(X_{t+1}=0, Z_{t+1}=0\right)$. The world is said to be tetranomial.

More generality could be introduced by letting $m_{1}, m_{2}, m_{3}$ and $m_{4}$ change over time. We shall not need that. ${ }^{3}$

\footnotetext{
${ }^{3}$ Notice that, if the state variable is the price of a share of stock, we will effectively be covering the risk of derivatives written on the stock using a bond portfolio. Such techniques have been considered before in the literature. See, e.g., Jarrow and Madan [1995]. If the stock is traded at each point in transaction time, it could replace one of the bonds in the hedge portfolio.
} 
If they exist, the solutions to (9) across $t$ form a stochastic process adapted to $\left\{\mathcal{F}_{t}\right\}_{t \geq 0}$. The existence of solutions hinges critically on the dimensionality of the payoff space of the bonds. Because of the tetranomial nature of uncertainty and Lemma 2, the dimension of the payoff space of any set of bonds cannot be more than four. Hence, we can restrict our attention to the payoffs of sets of four bonds. Then, for (9) to have a solution, there must be a choice of four maturities such that the payoff space generated by the bonds has dimension four.

Due to a result of Harrison and Kreps [1979], an equivalent way of investigating whether derivatives can be priced by arbitrage is to verify whether there exists a set of four bonds such that their prices imply unique Arrow-Debreu securities prices for each of the four states. We shall not take that route here, although we rejoin this approach at the end of Section $8 .{ }^{4}$

When solutions exist to (9) for all derivatives, we call the economy dynamically complete. Otherwise, it is incomplete.

In our economy, trading takes place in transaction time. The hedging equations in (9) are based on the possibility to rebalance the hedge portfolio in transaction time. What if we restrict our attention to rebalancing in calendar time? This would mean that if at time $t$, calendar time increases to $\xi$ and a position is established at that point, it can be changed only when calendar time augments to $(\xi+1)$, i.e., at the earliest $\tau>t$ for which $X_{\tau}=1$. The payoff space generated by this rebalancing restriction becomes very complex. It has a countably infinite number of possible outcomes. The change in the state variable over calendar period $(\xi, \xi+1)$, for instance, could be any element in the following list:

$$
s_{\xi+1}^{*}-s_{\xi}^{*}=\left\{\begin{array}{l}
s_{\xi}^{*}(u-1), \\
s_{\xi}^{*}(d-1), \\
s_{\xi}^{*}\left(u^{2}-1\right), \\
s_{\xi}^{*}(u d-1), \\
s_{\xi}^{*}\left(d^{2}-1\right) \\
s_{\xi}^{*}\left(u^{3}-1\right), \\
s_{\xi}^{*}\left(u^{2} d-1\right) \\
\cdots
\end{array}\right.
$$

It is clear that derivatives cannot be priced by arbitrage if only a finite number of bonds is available. Consequently, when trading is restricted to calendar time, the economy is incomplete.

Before we turn to a study of conditions for our economy to be dynamically complete (when trading takes place in transaction time), it is good to discuss first some basic restrictions on derivative prices which would hold even in an incomplete economy. These

\footnotetext{
${ }^{4}$ The two approaches are, however, not entirely equivalent. The Definition considers only a single derivative. Equivalence requires that solutions exist to (9) for all derivatives that one could possibly write. Later, we shall give an example where one derivative can be priced by arbitrage, but others may not. See Section 7.
} 
restrictions should be familiar from standard options analysis, but it is not clear that they continue to hold in our economy.

\section{General No-Arbitrage Restrictions}

Merton [1973] proves a set of restrictions on the pricing of put and call options written on a traded state variable. The analysis is entirely in calendar time and makes heavy use of the existence of a risk-free asset. In our economies, (i) trade takes place in transaction time, not calendar time; (ii) the relation between transaction time and calendar time is stochastic; (iii) there may not be a transaction-time risk-free asset; (iv) the state variable may not be traded.

Let us discard (iv) for the purpose of this section. In other words, we shall assume that the state variable is traded, and, to facilitate cross-reference to Merton's analysis, we shall refer to it as the "stock." Notice also that (iii) ought not be a problem if there exist (calendar-time) risk-free bonds that mature at the same moment in transaction time (as Merton implicitly did).

Problems may emerge, however, because the time elapsed till the next calendar-time tick may be very large. In other words, (i) and (ii) are the major hurdle. This is easiest to see with an example.

Translate into transaction time Merton's result that, for a European call with exercise price $k$ and maturity $m$,

$$
c_{t}^{m} \geq s_{t}-k B_{t}^{m} .
$$

This restriction on the call price obtains from considering the payoff on the following two static portfolios:

$\mathcal{P}_{1}$ : Purchase one unit of the stock;

$\mathcal{P}_{2}$ : Purchase one call and $k$ bonds.

(The zero-coupon bonds in $\mathcal{P}_{2}$ should carry the same maturity as the call.) At maturity of the call, say, at $t=t^{*}$, the payoff on $\mathcal{P}_{1}$ equals $s_{t^{*}}$, whereas that on $\mathcal{P}_{2}$ equals $\max \left(k, s_{t^{*}}\right)$. Hence, the payoff on the second position is always at least as large as that on the first one. If $t^{*}$ is known and finite, (11) immediately obtains. if

In our case, however, $t^{*}$ is random and possibly infinite (it is a stopping time). Even

$$
\lim _{T \rightarrow \infty} P\left\{t^{*}>T\right\}=0,
$$

(11) may not hold. The answer depends on how the arbitrageur discounts events in the future. $b_{t}$, the price of the one-transaction-period zero coupon bond, provides clues. We should immediately point out that $b_{t}$ may not be unique; if so, we take it to be the shadow price of this bond for the arbitrageur who contemplates exploiting the potential arbitrage caused by violation of (11). If $b_{t}<1$, the arbitrageur explicitly discounts in 
transaction time. If $b_{t}=1$, passage of transaction time is not discounted (this does not exclude the arbitrageur's discounting events in calendar time).

First, consider the case $b_{t}<1$. The arbitrageur discounts events in transaction time, and, hence, passage of transaction time must be dealt with explicitly. To simplify matters, take $m=1$. $t^{*}$ then becomes the first date in transaction time such that $X_{t^{*}}=1$. Now take a large, finite $T$. In states of the world where $t^{*} \leq T$, the previous analysis is correct. Otherwise, all one can say is that, at $T, \mathcal{P}_{1}$ pays $s_{T}$ and $\mathcal{P}_{2}$ pays $c_{T}^{1}-k B_{T}^{1}$.

It may very well be that $s_{T}>c_{T}^{1}-k B_{T}^{1}$ on the set of outcomes where $t^{*}>T$. As one increases $T, P\left\{t^{*}>T\right\}$ may decrease to zero, but $s_{T}-c_{T}^{1}-k B_{T}^{1}$ may increase without bound. The result is that investors (risk-averse ones in the first place) may not perceive an arbitrage opportunity even when initially $c_{t}^{1}<s_{t}-k B_{t}^{1}$.

To see how $s_{T}-c_{T}^{1}-k B_{T}^{1}$ could increase without bound in the absence of arbitrage opporunities, note that the result in Lemma 2 applies to call prices as well. This means that the Arrow-Debreu state prices that are consistent with bond prices ought to price an option as well. If state prices happen to be constant (the assumption merely simplifies the argument), we imply the following time- $T$ call price when $X_{T}=0$ and $Z_{T}=Z$ :

$$
\begin{aligned}
c_{T}^{1}\left(0, Z, \mathcal{F}_{T-1}\right) \\
=\quad q^{1,1} \max \left(0, S_{T} u-k\right)+q^{1,0} \max \left(0, S_{T} d-k\right) \\
\quad+q^{0,1} c_{T+1}^{1}\left(0,1, \mathcal{F}_{T}\right)+q^{0,0} c_{T+1}^{1}\left(0,0, \mathcal{F}_{T}\right) .
\end{aligned}
$$

Let us investigate the feasibility of solutions of the form

$$
c_{T}^{1}\left(0, Z, \mathcal{F}_{T-1}\right)=\alpha_{T}\left(0, Z, \mathcal{F}_{T-1}\right) s_{T},
$$

where:

$$
\alpha_{T}\left(0, Z, \mathcal{F}_{T-1}\right)<\rho<1 .
$$

If such solutions are feasible, boundedness of $B_{T}^{1}$ immediately implies that $s_{T}-c_{T}^{1}-k B_{T}^{1}$ increases with $s_{T}$ without bound.

Substitution of the suggested solution into (12) reveals that $\alpha_{T}$ ought to satisfy the following recursion:

$$
\begin{aligned}
\alpha_{T}\left(0, Z, \mathcal{F}_{T-1}\right)= & \left(q^{1,1} \frac{s_{T} u-k}{s_{T} u}+q^{0,1} \alpha_{T+1}\left(0,1, \mathcal{F}_{T}\right)\right) u \\
& +\left(q^{1,0} \frac{s_{T} d-k}{s_{T} d}+q^{0,0} \alpha_{T+1}\left(0,0, \mathcal{F}_{T}\right)\right) d
\end{aligned}
$$

For $s_{T}$ large $\left(k / s_{T} \approx 0\right)$, one solution is: $\alpha_{T}\left(0, Z, \mathcal{F}_{T-1}\right)=1$. But a solution where

$$
\alpha_{T}\left(0, Z, \mathcal{F}_{T-1}\right)<\rho<1
$$

is not infeasible. This is best seen by considering solutions where

$$
\alpha_{T}\left(0, Z, \mathcal{F}_{T-1}\right)=\alpha_{T+1}\left(0,1, \mathcal{F}_{T}\right)=\alpha
$$


For such solutions,

$$
\alpha=\frac{q^{1,1} u+q^{1,0} d}{1-\left(q^{0,1} u+q^{0,0} d\right)}
$$

which could very well be below some $\rho<1 .^{5}$

Now consider the second case, where $b_{t}=1$. Investors will then wait till the next calendar-time tick, no matter how far in the future. Essentially, investors can afford to ignore the number of transactions between two calendar-time ticks. As a result, the standard analysis will obtain: $\mathcal{P}_{2}$ always pays at least as much as $\mathcal{P}_{1}$, and, hence,

$$
c_{t}^{m} \geq s_{t}-k B_{t}^{m} .
$$

The first case, where $b_{t}<1$, is to be interpreted as one where there is an opportunity cost to transacting. In the case of arbitrage positions, such as a long position in $\mathcal{P}_{1}$ and a short position in $\mathcal{P}_{2}$, the investor incurs costs as the number of transactions increase before the position is unwound. This could be due, for instance, to increased margin requirement in the face of increases in volatility induced by the transactions. ${ }^{6}$ In other words, whenever there is an opportunity cost to not transacting, the first analysis is the right one and the restriction in (11) does not obtain. This seems particularly relevant for arbitrageurs who must tie scarce capital when attempting to exploit a perceived arbitrage opportunity.

Virtually all of Merton's option pricing restrictions are invalid in our economy when $b_{t}<1$. This includes well-known results such as put-call parity, which obtains as a simple extension of (11). The relevance of our theoretical analysis receives support from the empirical documentation of frequent violations of European put-call parity in, e.g., Kamara and Miller [1995].

Only the American feature of many exchange-traded options may force their prices to always behave according to Merton's restrictions (those, of course, that specifically pertain to American options). But notice that most exchange-traded options cannot (or will not) be exercised at more than one point in, say, a calendar-time day, e.g., the market's close. Interpreting our calendar-time ticks as the points in transaction time that the market closes, it becomes clear that our analysis of arbitrage pricing restrictions on European options is relevant for many exchange-traded American options as well.

\footnotetext{
${ }^{5}$ The feasibility of (13) implies that it is not necessarily true that $c_{T}^{1} / s_{T} \uparrow 1$ as $k / s_{T} \downarrow 0$.

${ }^{6}$ In practice, margin calls occur in calendar time. Nevertheless, they are triggered by volatility, and, hence, if transactions and volatility are related (as in this paper), by the (random) number of transactions between two calendar-time ticks. Of course, if the option's calendar-time maturity is 1, there will not be any margin calls anymore. If the maturity is more than $1(m>1)$, there may still be margin calls, and our analysis becomes relevant.
} 


\section{Necessary Conditions For Pricing By Arbitrage}

¿From the discussion in the previous section, one would conclude that derivatives prices may hardly be restricted. Because of the simple tetranomial stochastic structure of the economy, however, dynamic arbitrage arguments may provide restrictions where static arguments as in Merton [1973] do not.

In Section 2, we pointed out that market completeness, and, hence, the possibility to dynamically hedge derivative payoffs and price derivatives using arbitrage arguments, depend crucially on the dimension of the payoff space generated by the calendar-time bonds.

Some notation. Let $\bar{P}_{t}^{m}$ be the vector of payoffs across states at $t+1$ for a bond with maturity $m$ (as of time $t$ ).

$$
\bar{P}_{t}^{m}=\left[\begin{array}{l}
B_{t+1}^{m-1}\left(1,1, \mathcal{F}_{t}\right) \\
B_{t+1}^{m-1}\left(1,0, \mathcal{F}_{t}\right) \\
B_{t+1}^{m}\left(0,1, \mathcal{F}_{t}\right) \\
B_{t+1}^{m}\left(0,0, \mathcal{F}_{t}\right)
\end{array}\right]
$$

(The first two entries correspond to the states $\left(X_{t+1}=1, Z_{t+1}=1\right)$ and $\left(X_{t+1}=1, Z_{t+1}=\right.$ $0)$, respectively; the last two entries correspond to the states $\left(X_{t+1}=0, Z_{t+1}=1\right)$ and $\left(X_{t+1}=0, Z_{t+1}=0\right)$, respectively. $)$

So, to determine the completeness of the markets, the dimension of the space spanned by $\left\{\bar{P}_{t}^{m}\right\}_{m=m_{1}, m_{2}, m_{3}, m_{4}}$ is critical. This dimension is equal to the rank of the matrix $\bar{P}_{t}$, where

$$
\bar{P}_{t}=\left[\bar{P}_{t}^{m_{1}} \bar{P}_{t}^{m_{2}} \bar{P}_{t}^{m_{3}} \bar{P}_{t}^{m_{4}}\right]
$$

Let $r(A)$ denote the rank of a matrix $A$.

Define the $m$-period interest rate (yield on the $m$-period zero-coupon bond):

$$
y_{t}^{m}=\frac{1}{B_{t}^{m}}-1
$$

Our first fundamental result:

Theorem 3 For derivatives to be priced by arbitrage, interest rates $y_{t}^{m}$ must be stochastic.

Proof: Suppose the contrary. Then there are (deterministic) sequences $\left\{B_{t}^{o, m}\right\}_{t}($ all $m)$ such that

$$
\bar{P}_{t}^{m}=\left[\begin{array}{l}
B_{t+1}^{o, m-1} \\
B_{t+1}^{o, m-1} \\
B_{t+1}^{o, m} \\
B_{t+1}^{o, m}
\end{array}\right]
$$


Notice:

$$
\bar{P}_{t}^{m}=\left[\begin{array}{l}
1 \\
1 \\
0 \\
0
\end{array}\right] B_{t+1}^{o, m-1}+\left[\begin{array}{l}
0 \\
0 \\
1 \\
1
\end{array}\right] B_{t+1}^{o, m}
$$

Hence, $r\left(\bar{P}_{t}\right) \leq 2$, i.e., the dimension of the payoff space generated by $\bar{P}_{t}^{m}(m=$ $\left.m_{1}, m_{2}, m_{3}, m_{4}\right)$ is less than or equal to 2 . This is insufficient to span all possible outcomes across states.

When interest rates are stochastic, they could still be deterministic when recorded in calendar time. This means: the sequence

$$
\left\{y_{t(\xi)}^{m}\right\}_{\xi=0,1,2, \ldots}
$$

is deterministic $\left(t(\xi)=\min \left\{t: \xi_{t}=\xi\right\}\right)$. We now show that this sequence must not be constant for markets to be complete.

Theorem 4 For derivatives to be priced by arbitrage, interest rates must not be constant when recorded in calendar time.

Proof: Suppose the contrary. Then there are constants $B^{o, m-1}($ all $m>0)$ such that

$$
\bar{P}_{t}^{m}=\left[\begin{array}{l}
B^{o, m-1} \\
B^{o, m-1} \\
B_{t+1}^{m}\left(0,1, \mathcal{F}_{t}\right) \\
B_{t+1}^{m}\left(0,0, \mathcal{F}_{t}\right)
\end{array}\right]
$$

This means:

$$
\bar{P}_{t}=\left[\begin{array}{llll}
B^{o, m_{1}-1} & B^{o, m_{2}-1} & B^{o, m_{3}-1} & B^{o, m_{4}-1} \\
B^{o, m_{1}-1} & B^{o, m_{2}-1} & B^{o, m_{3}-1} & B^{o, m_{4}-1} \\
B_{t+1}^{m_{1}}\left(0,1, \mathcal{F}_{t}\right) & B_{t+1}^{m_{2}}\left(0,1, \mathcal{F}_{t}\right) & B_{t+1}^{m_{3}}\left(0,1, \mathcal{F}_{t}\right) & B_{t+1}^{m_{4}}\left(0,1, \mathcal{F}_{t}\right) \\
B_{t+1}^{m_{1}}\left(0,0, \mathcal{F}_{t}\right) & B_{t+1}^{m_{2}}\left(0,0, \mathcal{F}_{t}\right) & B_{t+1}^{m_{3}}\left(0,0, \mathcal{F}_{t}\right) & B_{t+1}^{m_{4}}\left(0,0, \mathcal{F}_{t}\right)
\end{array}\right]
$$

The first two rows are clearly colinear. Hence, $r\left(\bar{P}_{t}\right) \leq 3$. Consider the third and fourth rows. From Lemma 2, we know that there must exist $q_{t+1}^{X, Z}\left(0,1, \mathcal{F}_{t}\right)$ and $q_{t+1}^{X, Z}\left(0,0, \mathcal{F}_{t}\right)$ $(X=0,1 ; Z=0,1)$, such that:

$$
\left[\begin{array}{l}
B_{t+1}^{m_{1}}\left(0, Z, \mathcal{F}_{t}\right) \\
B_{t+1}^{m_{2}}\left(0, Z, \mathcal{F}_{t}\right) \\
B_{t+1}^{m_{3}}\left(0, Z, \mathcal{F}_{t}\right) \\
B_{t+1}^{m_{4}}\left(0, Z, \mathcal{F}_{t}\right)
\end{array}\right]=\left[\bar{P}_{t+1}\left(0, Z, \mathcal{F}_{t}\right)\right]^{\prime}\left[\begin{array}{l}
q_{t+1}^{1,1}\left(0, Z, \mathcal{F}_{t}\right) \\
q_{t+1}^{1,0}\left(0, Z, \mathcal{F}_{t}\right) \\
q_{t+1}^{0,1}\left(0, Z, \mathcal{F}_{t}\right) \\
q_{t+1}^{0,0}\left(0, Z, \mathcal{F}_{t}\right)
\end{array}\right]
$$

$(Z=0,1)$. We made explicit the dependence of $\bar{P}_{t+1}$ on $\left(X_{t+1}, Z_{t+1}, \mathcal{F}_{t}\right)$. Recursive substitution of the last two rows of $\bar{P}_{t+1}\left(0, Z, \mathcal{F}_{t}\right)$, using the fact that $q_{t}^{X, Z}<1$ (all $t$ ), 
and focusing on the stationary solution of the resulting difference equation, produces:

$$
\left[\begin{array}{c}
B_{t+1}^{m_{1}}\left(0, Z, \mathcal{F}_{t}\right) \\
B_{t+1}^{m_{2}}\left(0, Z, \mathcal{F}_{t}\right) \\
B_{t+1}^{m_{3}}\left(0, Z, \mathcal{F}_{t}\right) \\
B_{t+1}^{m_{4}}\left(0, Z, \mathcal{F}_{t}\right)
\end{array}\right]=\left[\begin{array}{c}
B^{o, m_{1}-1} \\
B^{o, m_{2}-1} \\
B^{o, m_{3}-1} \\
B^{o, m_{4}-1}
\end{array}\right] a_{t+1}^{Z}
$$

for some scalar random variable $a_{t+1}^{Z}$, measurable in $\mathcal{F}_{t+1}(Z=0,1)$. Hence, the third and fourth rows of $\bar{P}_{t}$ are colinear with the first and second ones. In other words, $r\left(\bar{P}_{t}\right)=1$, and markets are incomplete.

Remark 1: Even if not all derivatives can be priced by arbitrage if the conditions in Theorems 3 and 4 are violated, some may still be priced as such, if their payoff vector lies in a lower-dimensional space (i.e., with dimension strictly less than 4).

Remark 2: In proving Theorem 4, we made use of our restriction to consider only economies with non-explosive bond prices. For bubble economies, where bond prices may explode between calendar-time ticks, Theorem 4 does not obtain.

\section{These Conditions Are Not Sufficient}

We now consider an example that illustrates how the conditions in Theorems 3 and 4 are not sufficient.

We start from the specification of a process of Arrow-Debreu securities prices and will derive the corresponding bond price processes. Then we show that the payoff space generated by a choice of four bonds is lower-dimensional. Since the dimension is even less than or equal to two, markets cannot be complete.

Since markets will be shown to be incomplete, our specifying a process of ArrowDebreu securities from which to derive bond price processes essentially corresponds to picking an investor, observing her shadow prices for the Arrow-Debreu securities, and deducing what bond price processes must have looked like for them to be consistent with these shadow prices.

Let

$$
q_{t}^{X, Z}\left(X_{t}, Z_{t}, \mathcal{F}_{t-1}\right)=q^{X, Z}\left(X_{t}, Z_{t}\right)
$$

i.e., the state prices are (stationary) functions of only $X_{t}$ and $Z_{t}$.

Define

$$
Q=\left[\begin{array}{llll}
q^{1,1}(1,1) & q^{1,0}(1,1) & q^{0,1}(1,1) & q^{0,0}(1,1) \\
q^{1,1}(1,0) & q^{1,0}(1,0) & q^{0,1}(1,0) & q^{0,0}(1,0) \\
q^{1,1}(0,1) & q^{1,0}(0,1) & q^{0,1}(0,1) & q^{0,0}(0,1) \\
q^{1,1}(0,0) & q^{1,0}(0,0) & q^{0,1}(0,0) & q^{0,0}(0,0)
\end{array}\right]
$$


When normalized with the (shadow) prices of the transaction-time riskfree bond, $Q$ becomes a risk-neutral transition probability matrix from states at time $t$ to states at time $t+1$.

Assumption 6.1: Assume $Q$ is of full rank. Assume also:

$$
\begin{aligned}
& q^{0,1}(1,1)+q^{0,1}(1,0)+q^{0,1}(0,1)+q^{0,1}(0,0)<1 \\
& q^{0,0}(1,1)+q^{0,0}(1,0)+q^{0,0}(0,1)+q^{0,0}(0,0)<1
\end{aligned}
$$

It should be noted that this, it is not sufficient that $b_{t}<1$, all $t$. We shall consider only the stationary bond price processes consistent with these state prices. Hence,

$$
B_{t}^{m}\left(X_{t}, Z_{t}, \mathcal{F}_{t-1}\right)=B^{m}\left(X_{t}, Z_{t}\right) .
$$

Define, for $m \geq 0$,

$$
\bar{B}^{m}=\left[\begin{array}{l}
B^{m}(1,1) \\
B^{m}(1,0) \\
B^{m}(0,1) \\
B^{m}(0,0)
\end{array}\right] .
$$

As in Section 5, define, for $m>0$,

$$
\bar{P}^{m}=\left[\begin{array}{l}
B^{m-1}(1,1) \\
B^{m-1}(1,0) \\
B^{m}(0,1) \\
B^{m}(0,0)
\end{array}\right] .
$$

$\bar{P}^{m}$ is the vector of payoffs across states generated by a bond with maturity $m$. It depends neither on $t$ nor on $X_{t}$ or $Z_{t}$. Define:

$$
\bar{P}=\left[\bar{P}^{m_{1}} \bar{P}^{m_{2}} \bar{P}^{m_{3}} \bar{P}^{m_{4}}\right] .
$$

As before, the rank of $\bar{P}$ is crucial in determining market completeness.

We have a sequence of Lemmas which facilitate the proof of the main result (Theorem 9).

Lemma 5 For $m \geq 1$,

$$
\bar{B}^{m}=\left(I_{4}-\beta\right)^{-1} \alpha \bar{B}^{m-1},
$$

where

$$
\beta=\left[\begin{array}{llll}
0 & 0 & Q \cdot, 3 & Q \cdot, 4
\end{array}\right]
$$

$\left(Q_{\cdot, j}\right.$ denotes the $j$ th column of $\left.Q\right)$, and

$$
\alpha=\left[\begin{array}{llll}
Q_{\cdot, 1} & Q_{\cdot, 2} & 0 & 0
\end{array}\right] .
$$


(Proof: see Appendix.)

Let $\operatorname{dim}(F)$ denote the dimension of the vector space $F$.

\section{Corollary 6}

$$
\operatorname{dim}\left(\operatorname{span}\left\{\bar{B}^{m}, m=m_{1}, m_{2}, m_{3}, m_{4}\right\}\right) \leq 2 .
$$

Proof: $\bar{B}^{m}, m=m_{1}, m_{2}, m_{3}, m_{4}$, are linear combinations of the columns of $\left(I_{4}-\beta\right)^{-1} \alpha$. Since

$$
r\left(\left(I_{4}-\beta\right)^{-1} \alpha\right) \leq \min \left(r\left(\left(I_{4}-\beta\right)^{-1}\right), r(\alpha)\right)=2,
$$

the span generated by these vectors is at most of dimension 2 .

The following is a result that we do not really need for Theorem 9, but is nevertheless interesting on its own.

Lemma $7\left(I_{4}-\beta\right)^{-1} \alpha$ is not idempotent.

(Proof: see Appendix.) If this Lemma had not obtained, we would have, for any $m>1$ :

$$
\begin{aligned}
& \bar{B}^{m-1}=\left(I_{4}-\beta\right)^{-1} \alpha \bar{B}^{m-2}, \\
\bar{B}^{m} & =\left(I_{4}-\beta\right)^{-1} \alpha \bar{B}^{m-1} \\
& =\left(I_{4}-\beta\right)^{-1} \alpha\left(I_{4}-\beta\right)^{-1} \alpha \bar{B}^{m-2} \\
& =\left(I_{4}-\beta\right)^{-1} \alpha \bar{B}^{m-2} \\
& =\bar{B}^{m-1} .
\end{aligned}
$$

\section{Lemma 8}

$$
\bar{P}^{m}=\left[\gamma_{1}+\gamma_{0}\left(I_{4}-\beta\right)^{-1} \alpha\right] \bar{B}^{n-1},
$$

where

$$
\begin{aligned}
\gamma_{1} & =\left[\begin{array}{ll}
I_{2} & 0_{2 \times 2} \\
0_{2 \times 2} & 0_{2 \times 2}
\end{array}\right], \\
\gamma_{0} & =\left[\begin{array}{ll}
0_{2 \times 2} & 0_{2 \times 2} \\
0_{2 \times 2} & I_{2}
\end{array}\right] .
\end{aligned}
$$

$\left(I_{l}\right.$ denotes the $l \times l$ identity matrix; $0_{k \times l}$ denotes a $k \times l$ matrix of zeros).

Proof: Using Lemma 5,

$$
\begin{aligned}
\bar{P}^{m} & =\gamma_{1} \bar{B}^{m-1}+\gamma_{0} \bar{B}^{m} \\
& =\gamma_{1} \bar{B}^{m-1}+\gamma_{0}\left(I_{4}-\beta\right)^{-1} \alpha \bar{B}^{m-1} \\
& =\left[\gamma_{1}+\gamma_{0}\left(I_{4}-\beta\right)^{-1} \alpha\right] \bar{B}^{m-1} .
\end{aligned}
$$




\section{Theorem 9}

$$
r(\bar{P}) \leq 2 .
$$

Proof: $r(\bar{P})$ is the dimension of the space spanned by $\bar{P}^{m}, m=m_{1}, m_{2}, m_{3}, m_{4}$. The latter is obtained as a linear transformation of the space spanned by $\bar{B}^{m-1}, m=m_{1}, m_{2}, m_{3}, m_{4}$. This transformation is characterized by the matrix $\gamma_{1}+\gamma_{0}\left(I_{4}-\beta\right)^{-1} \alpha$, which is at best of rank 2. And the dimension of the space spanned by $\bar{B}^{m-1}, m=m_{1}, m_{2}, m_{3}, m_{4}$ is at most 2. Hence, the dimension of the space spanned by $\bar{P}^{m}, m=m_{1}, m_{2}, m_{3}, m_{4}$ is at most 2, i.e., $r(\bar{P}) \leq 2$.

Since the dimension of the payoff space generated by any four bonds is at most 2 , it is not generally possible to perfectly insure the risk of a derivative even if the hedge portfolio is rebalanced at every point in transaction time.

Notice, however, that interest rates are stochastic. In particular, they depend on $X_{t}$ and $Z_{t}$ :

$$
y_{t}^{m}\left(X_{t}, Z_{t}, \mathcal{F}_{t-1}\right)=y^{m}\left(X_{t}, Z_{t}\right)=\frac{1}{B^{m}\left(X_{t}, Z_{t}\right)}-1 .
$$

Consequently, we have here an example of a class of economies where: (i) derivatives cannot be priced by arbitrage, (ii) interest rates are stochastic.

\section{Independence Under A Risk-Neutral Probability}

We now consider the following case.

Assumption 7.1: There is a state price process for which $b_{t}=b<1$.

Assumption 7.2: $X_{t}$ and $Z_{t}$ are independent under the corresponding risk-neutral probability.

Assumption 7.3: the state variable is traded, and will be referred to as the "stock price."

As in the previous section, we again pick an investor in the economy and observe her risk-neutral probabilities (normalized shadow prices for Arrow-Debreu securities). Subsequently, we characterize the bond price processes which could have generated these. We then use this characterization to say something about market completeness. If markets turn out to be complete (which they do not), the economy only allows for the one choice of risk-neutral probabilities we initially made. 
To understand Assumption 7.2, let $\pi_{t}^{X, Z}$ denote the risk-neutral probability of $X_{t+1}=$ $X$ and $Z_{t+1}=Z$. It can be obtained from the state prices as follows:

$$
\pi_{t}^{X, Z}=\frac{q_{t}^{X, Z}}{b} .
$$

Define $p_{t}$ to be the marginal risk-neutral probability of $Z_{t+1}=1$ :

$$
p_{t}=\pi_{t}^{1,1}+\pi_{t}^{0,1} .
$$

Let $\Delta_{t}$ denote the marginal risk-neutral probability of $X_{t+1}=1$ :

$$
\Delta_{t}=\pi_{t}^{1,1}+\pi_{t}^{1,0} .
$$

We assume that the state variable is traded (Assumption 7.3). Hence, Lemma 2 restricts its evolution (see (4)). In this case,

$$
s_{t}=b\left(p_{t} s_{t} u+\left(1-p_{t}\right) s_{t} d\right),
$$

and we conclude that $p_{t}$ is a constant, to be denoted $p$. The independence assumption can now be stated as follows:

$$
\left\{\begin{array}{l}
\pi_{t}^{1,1}=p \Delta_{t}, \\
\pi_{t}^{1,0}=(1-p) \Delta_{t}, \\
\pi_{t}^{0,1}=p\left(1-\Delta_{t}\right), \\
\pi_{t}^{0,0}=(1-p)\left(1-\Delta_{t}\right) .
\end{array}\right.
$$

We add the following to these assumptions.

Assumption 7.4: $\Delta_{t}$ depends at most on $\left\{X_{t}, X_{t-1}, X_{t-2}, \ldots\right\}$.

Assumptions 7.1-7.4 impose the following structure on bond prices.

Lemma 10 Under Assumptions 7.1-7.4,

$$
B_{t}^{m}\left(X_{t}, 1, \mathcal{F}_{t-1}\right)=B_{t}^{m}\left(X_{t}, 0, \mathcal{F}_{t-1}\right) .
$$

(Proof: see Appendix.) Hence, bond price processes are binomial, driven only by the calendar-time tick process.

An immediate consequence is: the market is incomplete. This follows from Theorem 11.

Theorem 11 Under Assumptions 7.1-7.4,

$$
r\left(\bar{P}_{t}\right) \leq 2 .
$$


Proof: Consider the columns of $\bar{P}_{t}$ :

$$
\begin{aligned}
\bar{P}_{t}^{m} & =\left[\begin{array}{l}
B_{t+1}^{m-1}\left(1,1, \mathcal{F}_{t}\right) \\
B_{t+1}^{m-1}\left(1,0, \mathcal{F}_{t}\right) \\
B_{t+1}^{m}\left(0,1, \mathcal{F}_{t}\right) \\
B_{t+1}^{m}\left(0,0, \mathcal{F}_{t}\right)
\end{array}\right] \\
= & {\left[\begin{array}{l}
1 \\
1 \\
0 \\
0
\end{array}\right] B_{t+1}^{m-1}\left(1,1, \mathcal{F}_{t}\right)+\left[\begin{array}{l}
0 \\
0 \\
1 \\
1
\end{array}\right] B_{t+1}^{m}\left(0,1, \mathcal{F}_{t}\right), }
\end{aligned}
$$

$m=m_{1}, m_{2}, m_{3}, m_{4}$. Hence, $r\left(\bar{P}_{t}\right) \leq 2$.

One may still be able to price certain derivatives using arbitrage arguments. Here is an example. Consider a derivative with price $c_{t}^{m}$ for which the following is true.

Assumption 7.5: $c_{t+1}^{m}\left(0,1, \mathcal{F}_{t}\right)-c_{t+1}^{m-1}\left(1,1, \mathcal{F}_{t}\right)=c_{t+1}^{m}\left(0,0, \mathcal{F}_{t}\right)-c_{t+1}^{m-1}\left(1,0, \mathcal{F}_{t}\right)$

This assumption does not state that the term premium (incremental cost of longermaturity derivatives) is constant across levels of the stock price, because it compares values across states where $X_{t+1}=1$ and where $X_{t+1}=0$. Apply, however, this assumption to a call option with exercise price $k$. For such a derivative, we set:

$$
\begin{aligned}
c_{t+1}^{0}\left(1,1, \mathcal{F}_{t}\right) & =\max \left(0, s_{t} u-k\right), \\
c_{t+1}^{0}\left(1,0, \mathcal{F}_{t}\right) & =\max \left(0, s_{t} d-k\right) .
\end{aligned}
$$

Setting: $m=1$, Assumption 7.5 implies:

$$
c_{t+1}^{1}\left(0,1, \mathcal{F}_{t}\right)-\max \left(0, s_{t} u-k\right)=c_{t+1}^{1}\left(0,0, \mathcal{F}_{t}\right)-\max \left(0, s_{t} d-k\right),
$$

implying that the call's value for $X_{t+1}=0$ is obtained by adding a predetermined component to the immediate exercise value.

Assumption 7.5 causes redundancies in the system of equations (9) that represents the hedging problem. When we subsitute the stock for one of the bonds in the hedging portfolio, we obtain the following result.

Lemma 12 Under Assumptions 7.1-7.5, if $B_{t+1}^{1}\left(0,1, \mathcal{F}_{t}\right)$ is different from 1, the derivative's one-period payoff can be hedged with only the stock and a one-period bond.

Proof: see Appendix.

Solving (9) generates the following (recursive) formula.

Theorem 13 Under Assumptions 7.1-7.5, if $B_{t+1}^{1}\left(0,1, \mathcal{F}_{t}\right)$ is different from 1,

$$
\begin{aligned}
c_{t}^{m}\left(X_{t}, Z_{t}, \mathcal{F}_{t-1}\right) & \\
= & B_{t}^{1}\left(X_{t}, Z_{t}, \mathcal{F}_{t-1}\right) c_{t+1}^{m-1}\left(1,0, \mathcal{F}_{t}\right)+\alpha\left(X_{t}, Z_{t}, \mathcal{F}_{t-1}\right) \\
& \left(c_{t+1}^{m}\left(0,1, \mathcal{F}_{t}\right)-B_{t+1}^{1}\left(0,1, \mathcal{F}_{t}\right) c_{t+1}^{m-1}\left(1,0, \mathcal{F}_{t}\right)\right)
\end{aligned}
$$


where

$$
\alpha\left(X_{t}, Z_{t}, \mathcal{F}_{t-1}\right)=\frac{1-d B_{t}^{1}\left(X_{t}, Z_{t}, \mathcal{F}_{t-1}\right)}{u-d B_{t+1}^{1}\left(0,1, \mathcal{F}_{t}\right)}
$$

(Proof: see Appendix.)

Reconsider Assumption 7.5. Could it hold for a whole class of derivatives? Take, e.g., the class of call options generated by all possible exercise prices. At this point, it seems possible that all of them may satisfy Assumption 7.5 simultaneously. In particular, there do not even seem to be contradictions with the restrictions on option prices Merton [1973] derived on the basis of simple trading strategies executed in calendar time. Of course, as mentioned in Section 4, these restrictions need not hold in our context.

Theorem 13 therefore provides a reasonable option pricing formula one could work with in practice. It certainly is much more tractable than more general cases, to be discussed in the next section. It is attractive for another reason: only a single bond and the stock are needed to perfectly hedge the derivative's payoff (Lemma 12). In General, three bonds are needed, in addition to the stock.

Finally, let us turn back to Lemma 10. It is easy to prove Assumption 7.2 as a consequence of the claim in the Lemma. We state this as a theorem.

Theorem 14 If

$$
B_{t}^{m}\left(X_{t}, 1, \mathcal{F}_{t-1}\right)=B_{t}^{m}\left(X_{t}, 0, \mathcal{F}_{t-1}\right),
$$

then there exists a risk-neutral probability for which $X_{t}$ and $Z_{t}$ are independent.

This result is important. It provides a sufficient condition for there to exist a riskneutral probability such that the calendar-time tick process and the state variable process are independent. Hull and White [1987] have derived a stock option pricing formula under a risk-neutral probability measure for which the state variable (stock price) and its stochastic volatility were independent. Theorem 14 provides a sufficient condition on our bond price processes for the existence of a risk-neutral probability with the independence property. In other words, it describes a class of economies in our context for which the pricing technique popularized in Hull and White makes sense.

The latter also implies that Hull and White's technique could be used to generate an allternative option pricing formula to the one featured in Theorem 13. A derivation of this alternative formula would provide an occasion to explicitly compare the empirical success of two incomplete-markets option prices. There is one major difference between the two approaches: in Hull and White's, perfect replication remains impossible; in the approach that lead to Theorem 13, the option's payoff can be replicated using a portfolio of the stock and a one-period bond. 
The reference to Hull and White is not accidental. Hull and White investigated stock option pricing under stochastic volatility. As discussed in Section 2, our modeling procedure effectively introduces stochastic volatility in the stock price process when recorded in calendar time.

\section{A Class Of Stationary Economies With Complete Markets}

We now provide an example of a class of economies with complete markets, i.e., all derivatives can be priced by arbitrage. The economies will be stationary, in the sense that Arrow-Debreu securities prices (now unique) are time-invariant functions of stationary state variables that summarize relevant information.

The latter was already the case in the example of Section 6 . There:

$$
q_{t}^{X, Z}\left(X_{t}, Z_{t}, \mathcal{F}_{t-1}\right)=q^{X, Z}\left(X_{t}, Z_{t}\right)
$$

(in other words, the state variables were: $X_{t}$ and $Z_{t}$ ). We concluded that the markets were incomplete. We now enrich the set of state variables in a minimal way:

$$
q_{t}^{X, Z}\left(X_{t}, Z_{t}, \mathcal{F}_{t-1}\right)=q^{X, Z}\left(X_{t}, Z_{t}, X_{t-1}\right)
$$

The addition of $X_{t-1}$ as an argument of $q^{X, Z}$ introduces enough time-dependence on the state prices for markets to become complete. We shall need one important additional assumption, however. To understand what assumption is still missing, let us first investigate the class of economies where only (15) is imposed.

The discussion will clarify an important aspect of the notion of complete markets, namely, the crucial nature of the information flow. When Arrow-Debreu securities prices are only known to satisfy (15), the market is complete only conditional on certain information, i.e., conditional on certain histories of calendar-time ticks and stock price jumps. For other realizations, the market turns out to be incomplete. We can remedy the latter by introducing additional restrictions on the state prices.

We again follow the approach in Section 6: we pick an arbitrageur, observe the values she assigns to Arrow-Debreu securities and assume that they satisfy (15). We then investigate what class of bond prices processes is consistent with these valuations. We subsequently show that this class generates complete markets. A trivial consequence will be that the restriction in (15) is shared by all risk-neutral probability measures (it is trivial because there will be only one risk-neutral measure for each parametrization).

Define the matrices $Q^{i, j}$ :

$$
Q^{i, j}=\left[\begin{array}{ll}
q^{j, 1}(i, 1,1) & q^{j, 0}(i, 1,1) \\
q^{j, 1}(i, 0,1) & q^{j, 0}(i, 0,1) \\
q^{j, 1}(i, 1,0) & q^{j, 0}(i, 1,0) \\
q^{j, 1}(i, 0,0) & q^{j, 0}(i, 0,0)
\end{array}\right]
$$


for $i=0,1, j=0,1\left(i\right.$ indexes $X_{t} ; j$ indexes $\left.X_{t+1}\right)$. Assume: the $Q^{i, j}$ are all full-rank. Also: the columnsums of $Q^{1,0}$ and $Q^{0,0}$ are strictly less than 1 (we could do without this assumption; it is made to facilitate inversion of certain matrices).

As before, we want to consider only the stationary bond prices that are consistent with these state price processes. Hence,

$$
B_{t}^{m}\left(X_{t}, Z_{t}, \mathcal{F}_{t-1}\right)=B^{m}\left(X_{t}, Z_{t}, X_{t-1}\right) .
$$

Define, for $m \geq 0$,

$$
\bar{B}^{m}=\left[\begin{array}{c}
B^{m}(1,1,1) \\
B^{m}(1,0,1) \\
B^{m}(0,1,1) \\
B^{m}(0,0,1) \\
B^{m}(1,1,0) \\
B^{m}(1,0,0) \\
B^{m}(0,1,0) \\
B^{m}(0,0,0)
\end{array}\right]
$$

Define the time- $(t+1)$ payoff vector of a bond with maturity $m$ (at time $t ; m \geq 1$ ) if the time- $t$ state is $\left(X_{t}, Z_{t}, X_{t-1}\right)$ :

$$
\bar{P}^{m}\left(X_{t}, Z_{t}, X_{t-1}\right)=\left[\begin{array}{l}
B^{m-1}\left(1,1, X_{t}\right) \\
B^{m-1}\left(1,0, X_{t}\right) \\
B^{m}\left(0,1, X_{t}\right) \\
B^{m}\left(0,0, X_{t}\right)
\end{array}\right] .
$$

Notice:

$$
\begin{aligned}
& \bar{P}^{m}(1,1,1)=\bar{P}^{m}(1,0,1)=\bar{P}^{m}(1,1,0)=\bar{P}^{m}(1,0,0) ; \\
& \bar{P}^{m}(0,1,1)=\bar{P}^{m}(0,0,1)=\bar{P}^{m}(0,1,0)=\bar{P}^{m}(0,0,0) .
\end{aligned}
$$

For a choice of four maturities $m_{1}, m_{2}, m_{3}$ and $m_{4}$, define:

$$
\begin{aligned}
& \bar{P}\left(X_{t}, Z_{t}, X_{t-1}\right) \\
& \quad=\left[\bar{P}^{m_{1}}\left(X_{t}, Z_{t}, X_{t-1}\right) \bar{P}^{m_{2}}\left(X_{t}, Z_{t}, X_{t-1}\right) \bar{P}^{m_{3}}\left(X_{t}, Z_{t}, X_{t-1}\right) \bar{P}^{m_{4}}\left(X_{t}, Z_{t}, X_{t-1}\right)\right] .
\end{aligned}
$$

As before, $r\left(\bar{P}\left(X_{t}, Z_{t}, X_{t-1}\right)\right)$ (the rank of $\left.\bar{P}\left(X_{t}, Z_{t}, X_{t-1}\right)\right)$ is crucial in determining completeness of the markets.

Lemma 15 For $m \geq 1$,

$$
\bar{B}^{m}=\left(I_{8}-\beta\right)^{-1} \alpha \bar{B}^{m-1},
$$

where

$$
\beta=\left[\begin{array}{llllllll}
0 & 0 & q^{0,1}(1,1,1) & q^{0,0}(1,1,1) & 0 & 0 & 0 & 0 \\
0 & 0 & q^{0,1}(1,0,1) & q^{0,0}(1,0,1) & 0 & 0 & 0 & 0 \\
0 & 0 & 0 & 0 & 0 & 0 & q^{0,1}(0,1,1) & q^{0,0}(0,1,1) \\
0 & 0 & 0 & 0 & 0 & 0 & q^{0,1}(0,0,1) & q^{0,0}(0,0,1) \\
0 & 0 & q^{0,1}(1,1,0) & q^{0,0}(1,1,0) & 0 & 0 & 0 & 0 \\
0 & 0 & q^{0,1}(1,0,0) & q^{0,0}(1,0,0) & 0 & 0 & 0 & 0 \\
0 & 0 & 0 & 0 & 0 & 0 & q^{0,1}(0,1,0) & q^{0,0}(0,1,0) \\
0 & 0 & 0 & 0 & 0 & 0 & q^{0,1}(0,0,0) & q^{0,0}(0,0,0)
\end{array}\right]
$$


and

$$
\alpha=\left[\begin{array}{llllllll}
q^{1,1}(1,1,1) & q^{1,0}(1,1,1) & 0 & 0 & 0 & 0 & 0 & 0 \\
q^{1,1}(1,0,1) & q^{1,0}(1,0,1) & 0 & 0 & 0 & 0 & 0 & 0 \\
0 & 0 & 0 & 0 & q^{1,1}(0,1,1) & q^{1,0}(0,1,1) & 0 & 0 \\
0 & 0 & 0 & 0 & q^{1,1}(0,0,1) & q^{1,0}(0,0,1) & 0 & 0 \\
q^{1,1}(1,1,0) & q^{1,0}(1,1,0) & 0 & 0 & 0 & 0 & 0 & 0 \\
q^{1,1}(1,0,0) & q^{1,0}(1,0,0) & 0 & 0 & 0 & 0 & 0 & 0 \\
0 & 0 & 0 & 0 & q^{1,1}(0,1,0) & q^{1,0}(0,1,0) & 0 & 0 \\
0 & 0 & 0 & 0 & q^{1,1}(0,0,0) & q^{1,0}(0,0,0) & 0 & 0
\end{array}\right]
$$

(Proof: see Appendix.)

Lemma 16 For $m \geq 1$,

$$
\bar{P}^{m}\left(1, Z_{t}, X_{t-1}\right)=\gamma_{1} \bar{B}^{m-1}
$$

where

$$
\begin{gathered}
\gamma_{1}=\gamma_{11}+\gamma_{10}\left(I_{8}-\beta\right)^{-1} \alpha, \\
\gamma_{11}=\left[\begin{array}{ll}
I_{2} & 0_{2 \times 6} \\
0_{2 \times 2} & 0_{2 \times 6}
\end{array}\right], \\
\gamma_{10}=\left[\begin{array}{lll}
0_{2 \times 2} & 0_{2 \times 2} & 0_{2 \times 4} \\
0_{2 \times 2} & I_{2} & 0_{2 \times 4}
\end{array}\right] ; \\
\bar{P}^{m}\left(0, Z_{t}, X_{t-1}\right)=\gamma_{0} \bar{B}^{m-1},
\end{gathered}
$$

where

$$
\begin{gathered}
\gamma_{0}=\gamma_{01}+\gamma_{00}\left(I_{8}-\beta\right)^{-1} \alpha \\
\gamma_{01}=\left[\begin{array}{lll}
0_{2 \times 4} & I_{2} & 0_{2 \times 2} \\
0_{2 \times 4} & 0_{2 \times 2} & 0_{2 \times 2}
\end{array}\right], \\
\gamma_{00}=\left[\begin{array}{ll}
0_{2 \times 6} & 0_{2 \times 2} \\
0_{2 \times 6} & I_{2}
\end{array}\right]
\end{gathered}
$$

( $I_{l}$ denotes the $l \times l$ identity matrix; $0_{k \times l}$ denotes a $k \times l$ matrix of zeros).

Proof: Follows immediately from the definitions and Lemma 15.

Since $\bar{P}^{m}$, the payoff vector generated by a bond of maturity $m$, is a transformation of $\bar{B}^{m-1}$, we would need to show that this transformation is full rank. The dimension of the space spanned by a particular choice of four vectors $\bar{B}^{m-1}$ (we choose: $m=1,2,3,4$ ) will then be carried over to that spanned by the corresponding $\bar{P}^{m} \mathrm{~s}$. Unfortunately, whenever $X_{t}=0$, this transformation has only rank 2 . Whence the following Theorem.

\section{Theorem 17}

$$
\operatorname{dim}\left(\operatorname{span}\left\{\bar{P}^{m}\left(0, Z_{t}, X_{t-1}\right), m=1,2,3,4\right\}\right) \leq 2
$$


Proof: Inspect $\gamma_{0}$ (see Lemma 16): the bottom two rows of

$$
\left(I_{8}-\beta\right)^{-1} \alpha=\left(\sum_{l=0}^{\infty} \beta^{l}\right) \alpha
$$

(where $\beta^{0} \equiv I_{8}$ ) have zeros except in positions $(7,5),(7,6),(8,5)$ and $(8,6)$. When multiplied by $\gamma_{00}$, the resulting matrix only has nonzero elements in those columns where $\gamma_{01}$ does. This reveals that the rank of $\gamma_{0}$ is only two. Hence, whatever the dimension of span $\left\{\bar{B}^{m}, m=0,1,2,3\right\}, \bar{P}^{m}\left(0, X_{t}, Z_{t}\right), \cdot m=1,2,3,4$, forms at most a span of dimension 2 .

Conclusion: markets are incomplete whenever $X_{t}=0$. It can be shown, however, that, generically,

$$
\operatorname{dim}\left(\operatorname{span}\left\{\bar{P}^{m}\left(1, Z_{t}, X_{t-1}\right), m=1,2,3,4\right\}\right)=4 .
$$

In other words, markets are complete, only conditional on being in a state where $X_{t}=1$. Because they are incomplete otherwise, markets can only be called partially dynamically complete.

This illustrates that market completeness depends critically on the information filtration (we already pointed this out when discussing the effect on information filtrations of translations from transaction time to calendar time in Section 2). Conditional on certain information or histories, the markets may be revealed to be complete; conditioned on other information or histories, markets may be incomplete.

As a matter of fact, this partial incompleteness seems to be a general result. One can extend the state vector to include $X_{t-2}, X_{t-3}, \ldots, X_{t-T}$, i.e.,

$$
q_{t}^{X, Z}\left(X_{t}, Z_{t}, \mathcal{F}_{t-1}\right)=q^{X, Z}\left(X_{t}, Z_{t},\left\{X_{t-1}, X_{t-2}, X_{t-3}, \ldots, X_{t-T}\right\}\right),
$$

and still find that the markets are incomplete conditional on certain paths or histories. The paths where incompleteness obtains are those where $X_{t-T+1}=0$.

The incompleteness is caused by the fact that the future payoff of a bond with maturity $m$ depends only on $B^{m-1}(1,1,0)$ and $B^{m-1}(1,0,0)$ if $X_{t}=0$. In contrast, when $X_{t}=1$, this bond's future payoff depends on $B^{m-1}(1,1,1)$ and $B^{m-1}(1,0,1)$ as well.

To restore market completeness without adding any complexity or destroying the stationarity of the economies, we could add the following assumption.

Assumption 8.1:

$$
P\left\{X_{t+1}=1 \mid X_{t}=0\right\}=1 .
$$

Hence, $q^{0,1}\left(0, Z_{t}, X_{t-1}\right)=q^{0,0}\left(0, Z_{t}, X_{t-1}\right)=0$. This assumption does not overturn the property that the future payoff of a bond with maturity $m$ depends only on $B^{m-1}(1,1,0)$ and $B^{m-1}(1,0,0)$ if $X_{t}=0$; it does, however, reduce the number of future states from 
four to two $\left(Z_{t+1}\right.$ is either 0 or 1$)$. Because of this, just two bonds suffice to span all possible payoffs.

Redefine $\bar{B}^{m}, \bar{P}^{m}$ and $\bar{P}$ to reflect Assumption 8.1.

$$
\begin{gathered}
\bar{B}^{m}=\left[\begin{array}{l}
B^{m}(1,1,1) \\
B^{m}(1,0,1) \\
B^{m}(0,1,1) \\
B^{m}(0,0,1) \\
B^{m}(1,1,0) \\
B^{m}(1,0,0)
\end{array}\right] ; \\
\bar{P}^{m}\left(1, Z_{t}, X_{t-1}\right)=\left[\begin{array}{l}
B^{m-1}(1,1,1) \\
B^{m-1}(1,0,1) \\
B^{m}(0,1,1) \\
B^{m}(0,0,1)
\end{array}\right] ; \\
\bar{P}^{m}\left(0, Z_{t}, X_{t-1}\right)=\left[\begin{array}{l}
B^{m-1}(1,1,0) \\
B^{m-1}(1,0,0)
\end{array}\right] .
\end{gathered}
$$

(Notice that there are only two possible future states if $X_{t}=0$.) For a choice of four maturities $m_{1}, m_{2}, m_{3}$ and $m_{4}$, define:

$$
\begin{aligned}
& \bar{P}\left(X_{t}, Z_{t}, X_{t-1}\right)= \\
& \quad\left[\begin{array}{llll}
\bar{P}^{m_{1}}\left(X_{t}, Z_{t}, X_{t-1}\right) & \bar{P}^{m_{2}}\left(X_{t}, Z_{t}, X_{t-1}\right) & \bar{P}^{m_{3}}\left(X_{t}, Z_{t}, X_{t-1}\right) & \bar{P}^{m_{4}}\left(X_{t}, Z_{t}, X_{t-1}\right)
\end{array}\right] .
\end{aligned}
$$

Also, change the definitions of $Q^{0,1}$ and $Q^{0,0}$ :

$$
\begin{gathered}
Q^{0,1}=\left[\begin{array}{ll}
q^{1,1}(0,1,1) & q^{1,0}(0,1,1) \\
q^{1,1}(0,0,1) & q^{1,0}(0,0,1)
\end{array}\right], \\
Q^{0,0}=0 .
\end{gathered}
$$

$Q^{0,1}$ remains a full-rank matrix; $Q^{0,0}$, of course, now has zero rank.

Lemma 18 For $m \geq 1$,

$$
\bar{B}^{m}=\left(I_{6}+\beta\right) \alpha \bar{B}^{m-1}
$$

where

$$
\beta=\left[\begin{array}{llllll}
0 & 0 & q^{0,1}(1,1,1) & q^{0,0}(1,1,1) & 0 & 0 \\
0 & 0 & q^{0,1}(1,0,1) & q^{0,0}(1,0,1) & 0 & 0 \\
0 & 0 & 0 & 0 & 0 & 0 \\
0 & 0 & 0 & 0 & 0 & 0 \\
0 & 0 & q^{0,1}(1,1,0) & q^{0,0}(1,1,0) & 0 & 0 \\
0 & 0 & q^{0,1}(1,0,0) & q^{0,0}(1,0,0) & 0 & 0
\end{array}\right]
$$


and

$$
\alpha=\left[\begin{array}{llllll}
q^{1,1}(1,1,1) & q^{1,0}(1,1,1) & 0 & 0 & 0 & 0 \\
q^{1,1}(1,0,1) & q^{1,0}(1,0,1) & 0 & 0 & 0 & 0 \\
0 & 0 & 0 & 0 & q^{1,1}(0,1,1) & q^{1,0}(0,1,1) \\
0 & 0 & 0 & 0 & q^{1,1}(0,0,1) & q^{1,0}(0,0,1) \\
q^{1,1}(1,1,0) & q^{1,0}(1,1,0) & 0 & 0 & 0 & 0 \\
q^{1,1}(1,0,0) & q^{1,0}(1,0,0) & 0 & 0 & 0 & 0
\end{array}\right] .
$$

(Proof: see Appendix.)

Direct calculation reveals:

$$
=\left[\begin{array}{llllll}
q^{1,1}(1,1,1) & q^{1,0}(1,1,1) & 0 & 0 & q^{0,1}(1,1,1) q^{1,1}(0,1,1) & q^{0,1}(1,1,1) q^{1,0}(0,1,1) \\
& & & & +q^{0,0}(1,1,1) q^{1,1}(0,0,1) & +q^{0,0}(1,1,1) q^{1,0}(0,0,1) \\
q^{1,1}(1,0,1) & q^{1,0}(1,0,1) & 0 & 0 & q^{0,1}(1,0,1) q^{1,1}(0,1,1) & q^{0,1}(1,0,1) q^{1,0}(0,1,1) \\
& & & & +q^{0,0}(1,0,1) q^{1,1}(0,0,1) & +q^{0,0}(1,0,1) q^{1,0}(0,0,1) \\
0 & 0 & 0 & 0 & q^{1,1}(0,1,1) & q^{1,0}(0,1,1) \\
0 & 0 & 0 & 0 & q^{1,1}(0,0,1) & q^{1,0}(0,0,1) \\
q^{1,1}(1,1,0) & q^{1,0}(1,1,0) & 0 & 0 & q^{0,1}(1,1,0) q^{1,1}(0,1,1) & q^{0,1}(1,1,0) q^{1,0}(0,1,1) \\
& & & & +q^{0,0}(1,1,0) q^{1,1}(0,0,1) & +q^{0,0}(1,1,0) q^{1,0}(0,0,1) \\
q^{1,1}(1,0,0) & q^{1,0}(1,0,0) & 0 & 0 & q^{0,1}(1,0,0) q^{1,1}(0,1,1) & q^{0,1}(1,0,0) q^{1,0}(0,1,1) \\
& & & & +q^{0,0}(1,0,0) q^{1,1}(0,0,1) & +q^{0,0}(1,0,0) q^{1,0}(0,0,1)
\end{array}\right] .
$$

$\left(I_{6}+\beta\right) \alpha$ transforms $\bar{B}^{m-1}$ into $\bar{B}^{m}$. Hence, for span $\left\{\bar{B}^{m}, m=0,1,2,3\right\}$ to have dimension four, it is necessary that $\left(I_{6}+\beta\right) \alpha$ be of rank 4 . Inspection of (19) reveals that it will be, because of the assumptions on the matrices $Q^{i, j}(i=0,1 ; j=0,1)$. Conditions on the rank of $\left(I_{6}+\beta\right) \alpha$ alone are, however, not sufficient: for $m>0$, the $\bar{B}^{m}$ s do not obtain as rank-4 transformations of arbitrary vectors, but of the corresponding vectors $\bar{B}^{m-1}$ s. Nevertheless, we can prove the following.

Lemma 19 Generically,

$$
\operatorname{dim}\left(\operatorname{span}\left\{\bar{B}^{m}, m=0,1,2,3\right\}\right)=4 .
$$

(Proof: see Appendix.)

Now transform $\bar{B}^{m-1}$ into the payoff vectors, $\bar{P}^{m}\left(X_{t}, Z_{t}, X_{t-1}\right)$.

Lemma 20 For $m \geq 1$,

$$
\bar{P}^{m}\left(1, Z_{t}, X_{t-1}\right)=\gamma_{1} \bar{B}^{m-1}
$$

where

$$
\gamma_{1}=\gamma_{11}+\gamma_{10}\left(I_{6}+\beta\right) \alpha
$$




$$
\begin{gathered}
\gamma_{11}=\left[\begin{array}{ll}
I_{2} & 0_{2 \times 4} \\
0_{2 \times 2} & 0_{2 \times 4}
\end{array}\right], \\
\gamma_{10}=\left[\begin{array}{lll}
0_{2 \times 2} & 0_{2 \times 2} & 0_{2 \times 2} \\
0_{2 \times 2} & I_{2} & 0_{2 \times 2}
\end{array}\right] ; \\
P^{m}\left(0, Z_{t}, X_{t-1}\right)=\gamma_{0} \bar{B}^{m-1},
\end{gathered}
$$

where

$$
\gamma_{0}=\left[\begin{array}{ll}
0_{2 \times 4} & I_{2}
\end{array}\right]
$$

( $I_{l}$ denotes the $l \times l$ identity matrix; $0_{k \times l}$ denotes a $k \times l$ matrix of zeros).

Proof: Follows immediately from the definitions and Lemma 18.

Inspection of $\gamma_{0}$ and $\gamma_{1}$ reveal that these matrices (transformations) are always fullrank. Hence, we conclude:

\section{Theorem 21}

$$
\begin{aligned}
& r\left(\bar{P}\left(1, Z_{t}, X_{t-1}\right)\right)=4, \\
& r\left(\bar{P}\left(0, Z_{t}, X_{t-1}\right)\right)=2 .
\end{aligned}
$$

Since $r(\bar{P})$ need only be 2 when $X_{t}=0$ (there are only two possible future outcomes in that state), we now do obtain a dynamically complete market.

Summarizing: a stationary economy where state prices satisfy (15) is only partially complete. By introducing the assumption that $P\left\{X_{t+1}=1 \mid X_{t}=0\right\}=1$, we make the economy fully complete. In the more general case, where $q^{X, Z}$ depends on the history of calendar-time ticks up to lag $T$ (see (18)), we would merely need:

$$
P\left\{X_{t+1}=1 \mid X_{t}=X_{t-1}=\ldots=X_{t-T+1}=X_{t-T}=0\right\}=1 .
$$

$T$ can b e arbitrarily large, so we can still accomodate a rich set of stationary calendar-time tick processes.

We now rejoin an issue raised in Section 2: if markets are complete, one must be able to infer unique state prices from the prices of traded assets (in the present case, calendar-time bonds). How would one go about extracting such prices here?

In total, there are 20 state prices to be solved for. Using the prices of bonds with maturities 1,2,3 and 4, the state prices can be obtained from the following equations:

$$
\bar{B}^{m}=\beta \bar{B}^{m}+\alpha \bar{B}^{m-1},
$$


for $m=1,2,3,4$. In total, there are 24 equations, of which four will be redundant. One can collect nonredundant equations into one system:

$$
\bar{B}=\delta \bar{Q},
$$

where:

$$
\begin{aligned}
& \bar{Q}^{\prime} \\
& =\left[q^{1,1}(1,1,1) \quad q^{1,0}(1,1,1) \quad q^{0,1}(1,1,1) \quad q^{0,0}(1,1,1)\right. \\
& q^{1,1}(1,0,1) \quad q^{1,0}(1,0,1) \quad q^{0,1}(1,0,1) . q^{0,0}(1,0,1) \\
& q^{1,1}(1,1,0) \quad q^{1,0}(1,1,0) \quad q^{0,1}(1,1,0) \quad q^{0,0}(1,1,0) \\
& q^{1,1}(1,0,0) \quad q^{1,0}(1,0,0) \quad q^{0,1}(1,0,0) \quad q^{0,0}(1,0,0) \\
& \left.q^{1,1}(0,1,1) \quad q^{1,0}(0,1,1) \cdots q^{1,1}(0,0,1) \backsim q^{1,0}(0 ; 0,1)\right], \\
& \bar{B}^{\prime} \\
& =\left[\begin{array}{llll}
B^{1}(1,1,1) & B^{2}(1,1,1) \quad B^{3}(1,1,1) \quad B^{4}(1,1,1)
\end{array}\right. \\
& B^{1}(1,0,1) \quad B^{2}(1,0,1) \quad B^{3}(1,0,1) \quad B^{4}(1,0,1) \\
& B^{1}(1,1,0) \quad B^{2}(1,1,0) \quad B^{3}(1,1,0) \quad B^{4}(1,1,0) \\
& B^{1}(1,0,0) \quad B^{2}(1,0,0) \quad B^{3}(1,0,0) \quad B^{4}(1,0,0) \\
& \left.B^{1}(0,1,1) \quad B^{2}(0,1,1) \quad B^{1}(0,0,1) \quad B^{2}(0,0,1)\right] \text {, } \\
& \delta=\left[\begin{array}{llllll}
\bar{P}(1,1,1)^{\prime} & 0 & 0 & 0 & 0 & 0 \\
0 & \bar{P}(1,0,1)^{\prime} & 0 & 0 & 0 & 0 \\
0 & 0 & \bar{P}(1,1,0)^{\prime} & 0 & 0 & 0 \\
0 & 0 & 0 & \bar{P}(1,0,0)^{\prime} & 0 & 0 \\
0 & 0 & 0 & 0 & \bar{P}(0,1,1)^{\prime} & 0 \\
0 & 0 & 0 & 0 & 0 & \bar{P}(0,0,1)^{\prime}
\end{array}\right]
\end{aligned}
$$

where we restrict $\bar{P}(0,1,1)$ and $\bar{P}(0,0,1)$ to include only the payoff vectors for bonds with maturities 1 and 2 (hence, they are 2 by 2 matrices, instead of 2 by 4 ). Because of Theorem $21, \delta$ is a full-rank matrix. The (unique) state prices are obtained by inversion:

$$
\bar{Q}=\delta^{-1} \bar{B}
$$

Until now, we have followed a route where we first picked state price processes, determined consistent bond price processes, and then verified whether markets were complete. Using (20), we can now sketch the alternative, traditional route, where one posits, say, stationary bond price processes of the form $B^{m}\left(X_{t}, Z_{t}, X_{t-1}\right)$, and one verifies whether they: (i) are free of arbitrage opportunities, (ii) give rise to complete markets. If one excludes the states $\left(0, Z_{t}, 0\right)$ a priori, state prices must solve (20). If no strictly positive 
solution exists, the given bond price processes are inconsistent with (i), i.e., with absence of arbitrage opportunities. If such solutions do exist, there are no arbitrage opportunities. If the solution is unique, markets are complete, i.e., (ii) obtains. Our approach had the advantage that it generated bond price processes that automatically satisfied (i), so that we could focus on (ii).

\section{Conclusion}

This paper has begun to analyze the restrictions imposed by absence of arbitrage in an economy where arbitrageurs take decisions in transaction time but finite-maturity contracts bear (random) calendar-time expiration dates. Since portfolio rebalancing is possible but in transaction time, our approach is only natural. Yet the implications of it are profound: some of the simple arbitrage restrictions that obtain when decisions are taken in calendar time may fail in our world; simultaneously, new opportunities for dynamic hedging, and, hence, pricing by arbitrage, are offered. Among other things, arbitrage-based solutions become possible for the pricing of options written on stock whose price exhibits stochastic volatility when recorded in calendar time. Whence the title of this paper.

We mentioned that it was not innocuous to assume that there exists a transaction time (count of occasions) when it is possible to rebalance a hedge portfolio potentially consisting of multiple securities, and for which the state variable process is binomial. Realistically, this would require continuous trading in the hedge securities. In the present case, these were riskfree bonds with calendar-time maturities. It would be interesting to study an economy where bond trading is noncontinuous, and even asynchronous, so that not all the components of the hedge portfolio can be adjusted simultaneously. A continuously traded security (money?) will still be necessary, because it is difficult to see how transactions would technically be possible. After all, transactions are exchanges of one asset for another. 


\section{References}

Black, F. and M. Scholes, 1973, The Pricing of Options and Corporate Liabilities, Journal of Political Economy 81, 637-659.

Cox, J., J. Ingersoll, and S. Ross, 1985, "An Intertemporal General Equilibrium Model of Asset Prices," Econometrica 53, 363-384.

Clark, P., 1973, "A Subordinated Stochastic Process Model with Finite Variance for Speculative Prices," Econometrica 41, 135-156.

Delbaen, F. and H. Shirakawa, 1996, "Option Pricing for Constant Elasticity of Variance Model", Discussion Paper Department of Mathematics, ETH, Zurich.

Engle, R.F. and J.R. Russell, 1996, "Forecasting the Frequency of Changes in Quoted Foreign Exchange Prices with the ACD Model," Econometrica, forthcoming.

Geman, H. and M. Yor, 1993, "Bessel Processes, Asian Options, and Perpetuities," Mathematical Finance 3, 349-375.

Geman, H. and M. Yor, 1995, "Pricing and Hedging Double-Barrier Options: A Probabilistic Approach," Discussion Paper ESSEC, Paris.

Ghysels, E. and J. Jasiak, 1995, "Stochastic Volatility and Time Deformation: An Application to Trading Volume and Leverage Effects," Université de Montréal working paper.

Harris, L., 1986, "Cross-Security Tests of the Mixture of Distribution Hypothesis," Journal of Financial and Quantitative Analysis 21, 39-46.

Harris, L., 1987, "Transaction Data Tests of the Mixture of Distribution Hypothesis," Journal of Financial and Quantitative Analysis 22, 127-142.

Harrison, J.M. and D. Kreps, 1979. "Martingales and Arbitrage in Multiperiod Securities Markets," Journal of Economic Theory 20, 381-408.

Harrison, J.M. and S. R. Pliska, 1981, "Martingales and Stochastic Integrals in the Theory of Continuous Trading," Stochastic Processes and Their Applications 11, 215-260.

Heath, D., R. Jarrow and A. Morton, 1992, "Bond Pricing and the Term Structure of Interest Rates: A New Methodology for Contingent Claims Valuation," Econometrica $60,77-106$.

Ho, T.S. and S. Lee, 1986, "Term Structure Movements and Pricing Interest Rate Contingent Claims," Journal of Finance 41, 1011-1028.

Hull, J. and A. White, 1987, "The Pricing of Options on Assets with Stochastic Volatilities," Journal of Finance 3, 281-300. 
Jarrow, R. and D. Madan, 1995, "Option Pricing Using the Term Structure of Interest Rates to Hedge Systematic Discontinuities in Asset Returns," Mathematical Finance 5, 311-336.

Kamara, A. and T.W. Miller, 1995, "Daily and Intradaily Tests of European Put-Call Parity," Journal of Financial and Quantitative Analysis 30, 519-540.

Leblanc, B. and O. Scaillet, 1996, "Path-Dependent Options on Yields in the Affine Term Structure Model", Discussion Paper CREST, Paris.

Madan, D. and E. Seneta, 1990, "The V.G. Model for Share Market Returns," Journal of Business 63, 511-524.

Merton, R., 1973, "The Theory of Rational Option Pricing," Bell Journal of Economics and Management Science 4, 141-183.

Tauchen, G. and M. Pitts, 1983, "The Price Variability-Volume Relationship on Speculative Markets," Econometrica 51, 485-505. 


\section{Appendix}

\section{Proof of Theorem 1}

Under the maintained assumptions,

$$
\begin{aligned}
& E\left[\left(U_{\xi}^{*}\right)^{2} \mid \mathcal{G}_{\xi-1}\right] \\
& \quad=V\left[\left(U_{\xi}^{*}\right)^{2} \mid \mathcal{G}_{\xi-1}\right]+\left(E\left[U_{\xi}^{*} \mid \mathcal{G}_{\xi-1}\right]\right)^{2} .
\end{aligned}
$$

But,

$$
\begin{aligned}
& E {\left[U_{\xi}^{*} \mid \mathcal{G}_{\xi-1}\right] } \\
&= E\left[E\left[\sum_{\tau=t(\xi-1)}^{t(\xi)-1}\left(Z_{\tau+1}(\tilde{u}-\tilde{d})+\tilde{d}\right) \mid X_{t(\xi)}, X_{t(\xi)-1}, \ldots, X_{0} ; Z_{t(\xi-1)}, Z_{t(\xi-1)-1}, \ldots, Z_{0}\right]\right. \\
&\left.\mid \mathcal{G}_{\xi-1}\right] \\
&= 0 ; \\
& V\left[U_{\xi}^{*} \mid \mathcal{G}_{\xi-1}\right] \\
&=\quad V\left[E\left[U_{\xi}^{*} \mid X_{t(\xi)}, X_{t(\xi)-1}, \ldots, X_{0} ; Z_{t(\xi-1)}, Z_{t(\xi-1)-1}, \ldots, Z_{0}\right] \mid \mathcal{G}_{\xi-1}\right] \\
& \quad+E\left[V\left[U_{\xi}^{*} \mid X_{t(\xi)}, X_{t(\xi)-1}, \ldots, X_{0} ; Z_{t(\xi-1)}, Z_{t(\xi-1)-1}, \ldots, Z_{0}\right] \mid \mathcal{G}_{\xi-1}\right] \\
&=\quad E\left[V\left[U_{\xi}^{*} \mid X_{t(\xi)}, X_{t(\xi)-1}, \ldots, X_{0} ; Z_{t(\xi-1)}, Z_{t(\xi-1)-1}, \ldots, Z_{0}\right] \mid \mathcal{G}_{\xi-1}\right] \\
&=\quad E\left[V\left[\sum_{t(\xi)-1}\left(Z_{\tau+1}(\tilde{u}-\tilde{d})+\tilde{d}\right) \mid X_{t(\xi)}, X_{t(\xi)-1}, \ldots, X_{0} ; Z_{t(\xi-1)}, Z_{t(\xi-1)-1}, \ldots, Z_{0}\right]\right. \\
&\left.\quad \mid \mathcal{G}_{\xi-1}\right] \\
&=\quad \sigma^{2} E\left[t(\xi)-t(\xi-1) \mid \mathcal{G}_{\xi-1}\right],
\end{aligned}
$$

where $\sigma^{2}$ is the variance of $Z_{\tau+1}(\tilde{u}-\tilde{d})+\tilde{d}$.

\section{Proof of Lemma 2}

The existence of the $q_{t}^{X, Z}$ follows from Harrison and Kreps [1979]. The restriction that $0<q_{t}^{X, Z}<1$ follows from our assumptions that: (i) $0<P\left\{X_{t}=1\right\}<1,0<P\left\{Z_{t}=\right.$ $1\}<1$, and (ii) $b_{t}=q_{t}^{1,1}+q_{t}^{1,0}+q_{t}^{0,1}+q_{t}^{0,0} \leq 1$.

\section{Proof of Lemma 5}

The following obtains after writing out explicitly the result of Lemma 2:

$$
\bar{B}^{m}=\beta \bar{B}^{m}+\alpha \bar{B}^{m-1} .
$$


Hence,

$$
\left(I_{4}-\beta\right) \bar{B}^{m}=\alpha \bar{B}^{m-1} .
$$

Because of the assumed restriction in Assumption $6.1,\left(I_{4}-\beta\right)$ is invertible and its inverse equals $\sum_{l \geq 0} \beta^{l}$, where $\beta^{0} \equiv I_{4}$. Hence,

$$
\bar{B}^{m}=\left(I_{4}-\beta\right)^{-1} \alpha \bar{B}^{m-1} .
$$

\section{Proof of Lemma 7}

Because $Q$ is full rank, any matrix $A$ which satisfies:

$$
\alpha A=\alpha
$$

must have the identity 2 by 2 matrix in its Northwest corner. For $\left(I_{4}-\beta\right)^{-1} \alpha$ to be idempotent, it must be that:

$$
\left(I_{4}-\beta\right)^{-1} \alpha\left(I_{4}-\beta\right)^{-1} \alpha=\left(I_{4}-\beta\right)^{-1} \alpha .
$$

Rewriting, one obtains:

$$
\alpha\left(I_{4}-\beta\right)^{-1} \alpha=\alpha .
$$

For this to be possible, $\left(I_{4}-\beta\right)^{-1} \alpha$ must satisfy the restrictions on $A$ above. But, because of the assumed restriction on the columnsums of $Q$,

$$
\begin{aligned}
\left(I_{4}-\beta\right)^{-1} \alpha & =\sum_{l=0}^{\infty} \beta^{l} \alpha \\
& \geq \alpha
\end{aligned}
$$

$\left(\beta^{0} \equiv I_{4}\right)$. Since element $(1,2)$ of $\alpha$ is strictly positive, whereas the same element of $A$ must be zero, a contradiction is obtained.

\section{Proof of Lemma 10}

We make explicit the assumption that $\Delta_{t}$ depends only on the history of calendar-time ticks by writing:

$$
\Delta_{t}=\Delta_{t}\left(X_{t}, X_{t-1}, \ldots\right) .
$$

Taking first $m=1$, appealing to Lemma 2 , and focusing on the stationary solution,

$$
\begin{aligned}
& B_{t}^{1}\left(X_{t}, Z_{t}, \mathcal{F}_{t-1}\right) \\
&= b \Delta\left(X_{t}, X_{t-1}, \ldots\right) \\
&+b^{2}\left(1-\Delta\left(X_{t}, X_{t-1}, \ldots\right)\right) \Delta\left(X_{t+1}, X_{t}, \ldots\right) \\
&+b^{3}\left(1-\Delta\left(X_{t}, X_{t-1}, \ldots\right)\right)\left(1-\Delta\left(X_{t+1}, X_{t}, \ldots\right)\right) \Delta\left(X_{t+2}, X_{t+2}, \ldots\right) \\
& \ldots
\end{aligned}
$$


Clearly, $B_{t}^{1}\left(X_{t}, 1, \mathcal{F}_{t-1}\right)=B_{t}^{1}\left(X_{t}, 0, \mathcal{F}_{t-1}\right)$. Since $B_{t}^{2}\left(X_{t}, Z_{t}, \mathcal{F}_{t-1}\right)$ depends on the evolution of future one-period bond prices, and the latter are the same across $Z_{t}=0,1$, the same conclusion obtains for $m=2$. Iterating, one can prove the result for all $m$.

\section{Proof of Lemma 12}

Consider the system in (9). Subsitute the stock for the fourth bond. The system becomes:

$$
\begin{aligned}
c_{t+1}^{m-1}\left(1,1, \mathcal{F}_{t}\right)= & w_{t}^{s} s_{t} u+w_{t}^{1} B_{t+1}^{m_{1}-1}\left(1,1, \mathcal{F}_{t}\right) \\
& +w_{t}^{2} B_{t+1}^{m_{2}-1}\left(1,1, \mathcal{F}_{t}\right)+w_{t}^{3} B_{t+1}^{m_{3}-1}\left(1,1, \mathcal{F}_{t}\right) \\
c_{t+1}^{m-1}\left(1,0, \mathcal{F}_{t}\right)= & w_{t}^{s} s_{t} d+w_{t}^{1} B_{t+1}^{m_{1}-1}\left(1,0, \mathcal{F}_{t}\right) \\
& +w_{t}^{2} B_{t+1}^{m_{2}-1}\left(1,0, \mathcal{F}_{t}\right)+w_{t}^{3} B_{t+1}^{m_{3}-1}\left(1,0, \mathcal{F}_{t}\right) \\
c_{t+1}^{m}\left(0,1, \mathcal{F}_{t}\right)= & w_{t}^{s} s_{t} u+w_{t}^{1} B_{t+1}^{m_{1}}\left(0,1, \mathcal{F}_{t}\right) \\
& +w_{t}^{2} B_{t+1}^{m_{2}}\left(0,1, \mathcal{F}_{t}\right)+w_{t}^{3} B_{t+1}^{m_{3}}\left(0,1, \mathcal{F}_{t}\right) \\
c_{t+1}^{m}\left(0,0, \mathcal{F}_{t}\right)= & w_{t}^{s} s_{t} d+w_{t}^{1} B_{t+1}^{m_{1}}\left(0,0, \mathcal{F}_{t}\right) \\
& +w_{t}^{2} B_{t+1}^{m_{2}}\left(0,0, \mathcal{F}_{t}\right)+w_{t}^{3} B_{t+1}^{m_{3}}\left(0,0, \mathcal{F}_{t}\right)
\end{aligned}
$$

Now impose the result of Lemma 10 and observe that the second and third bonds are redundant. Assumption 7.5 will guarantee that there is no inconsistency, despite the redundancies. Hence, the system can be solved using just the stock and a one-period bond.

\section{Proof of Theorem 13}

Use the second and third equations in (21), take $m_{1}=1$ (allowed because of the assumption on $B_{t}^{1}$ ), and set the weights to the second and third bonds equal to zero. The solution is:

$$
\begin{aligned}
w_{t}^{s} & =\frac{c_{t+1}^{m}\left(0,1, \mathcal{F}_{t}\right)-c_{t+1}^{m-1}\left(1,0, \mathcal{F}_{t}\right) B_{t+1}^{1}\left(0,1, \mathcal{F}_{t}\right)}{s_{t} u-s_{t} d B_{t+1}^{1}\left(0,1, \mathcal{F}_{t}\right)} \\
w_{t}^{1} & =c_{t+1}^{m-1}\left(1,0, \mathcal{F}_{t}\right)-w_{t}^{s} s_{t} d .
\end{aligned}
$$

The result then obtains by setting:

$$
c_{t}^{m}\left(X_{t}, Z_{t}, \mathcal{F}_{t-1}\right)=w_{t}^{s} s_{t}+w_{t}^{1} B_{t}^{1}\left(X_{t}, Z_{t}, \mathcal{F}_{t-1}\right) .
$$




\section{Proof of Theorem 14}

According to (3), bond prices satisfy:

$$
\begin{aligned}
& B_{t}^{m}\left(X_{t}, Z_{t}, \mathcal{F}_{t-1}\right) \\
& \quad q_{t}^{1,1}\left(X_{t}, Z_{t}, \mathcal{F}_{t-1}\right) B_{t+1}^{m-1}\left(1,1, \mathcal{F}_{t}\right)+q_{t}^{1,0}\left(X_{t}, Z_{t}, \mathcal{F}_{t-1}\right) B_{t+1}^{m-1}\left(1,0, \mathcal{F}_{t}\right) \\
& \quad+q_{t}^{0,1}\left(X_{t}, Z_{t}, \mathcal{F}_{t-1}\right) B_{t+1}^{m}\left(0,1, \mathcal{F}_{t}\right)+q_{t}^{0,0}\left(X_{t}, Z_{t}, \mathcal{F}_{t-1}\right) B_{t+1}^{m}\left(0,0, \mathcal{F}_{t}\right) .
\end{aligned}
$$

Defining the risk-neutral probabilities as

$$
\pi_{t}^{X, Z}=\frac{q_{t}^{X, Z}}{b_{t}}
$$

and using the assumption that

$$
B_{t+1}^{m}\left(X_{t+1}, 1, \mathcal{F}_{t}\right)=B_{t+1}^{m}\left(X_{t+1}, 0, \mathcal{F}_{t}\right),
$$

we rewrite this as follows:

$$
\begin{aligned}
& B_{t}^{m}\left(X_{t}, Z_{t}, \mathcal{F}_{t-1}\right) \\
&=b_{t}\left(\left[\pi_{t}^{1,1}\left(X_{t}, Z_{t}, \mathcal{F}_{t-1}\right)+\pi_{t}^{1,0}\left(X_{t}, Z_{t}, \mathcal{F}_{t-1}\right)\right] B_{t+1}^{m-1}\left(1,1, \mathcal{F}_{t}\right)\right. \\
&\left.\quad+\left[\pi_{t}^{0,1}\left(X_{t}, Z_{t}, \mathcal{F}_{t-1}\right)+\pi_{t}^{0,0}\left(X_{t}, Z_{t}, \mathcal{F}_{t-1}\right)\right] B_{t+1}^{m}\left(0,1, \mathcal{F}_{t}\right)\right) .
\end{aligned}
$$

Retaining this equation for two bonds with differing maturities, and adding the requirement that

$$
\pi_{t}^{1,1}+\pi_{t}^{1,0}+\pi_{t}^{0,1}+\pi_{t}^{0,0}=1,
$$

one can rewrite these to become three linear, independent equations in the five unknowns $b_{t} \pi_{t}^{1,1}, b_{t} \pi_{t}^{1,0}, b_{t} \pi_{t}^{0,1}, b_{t} \pi_{t}^{0,0}$ and $b_{t}$. The solution set is nonempty and at least two-dimensional. We choose a solution so that the corresponding marginal risk-neutral probabilities $p_{t}$ and $\Delta_{t}$ satisfy the independence requirement:

$$
\left\{\begin{array}{l}
\pi_{t}^{1,1}=p_{t} \Delta_{t} \\
\pi_{t}^{1,0}=\left(1-p_{t}\right) \Delta_{t}, \\
\pi_{t}^{0,1}=p_{t}\left(1-\Delta_{t}\right) \\
\pi_{t}^{0,0}=\left(1-p_{t}\right)\left(1-\Delta_{t}\right)
\end{array}\right.
$$

(see (14)). The latter constitute four linear, independent equations if we take the unknowns to be the $\pi_{t}^{X, Z} \mathrm{~s}$ and $p_{t} \Delta_{t}$ and $\Delta_{t}$. When these equations are added, we obtain in total seven equations in seven unknowns. A solution exists and will generally be unique.

\section{Proof of Lemma 15}

Analogous to the proof of Lemma 5. 


\section{Proof of Lemma 18}

Analogous to the proof of Lemma 5. Also notice that in this case,

$$
\begin{aligned}
\left(I_{6}-\beta\right)^{-1} & =\sum_{l=0}^{\infty} \beta^{l} \\
& =\beta^{0}+\beta^{1} \\
& =I_{6}+\beta
\end{aligned}
$$

(where we again use: $\beta^{0} \equiv I_{6}$ ).

\section{Proof of Lemma 19}

First notice that, generically, there does not exist a scalar $g_{0}$ such that

$$
\bar{B}^{1}\left(=\left(I_{6}+\beta\right) \alpha \bar{B}^{0}\right)=g_{0} \bar{B}^{0} .
$$

This can best be seen by remembering that $\bar{B}^{0}$ is a vector with 1 in all positions, and, from (19), that the third and fourth elements of $\bar{B}^{1}$ will therefore be:

$$
\begin{aligned}
& q^{1,1}(0,1,1)+q^{1,0}(0,1,1), \\
& q^{1,1}(0,0,1)+q^{1,0}(0,0,1),
\end{aligned}
$$

respectively, i.e., the rowsums of $Q^{0,1}$. Generically, these rowsums will differ, and, hence, $\bar{B}^{1}$ cannot be written as $g_{0} \bar{B}^{0}$. Therefore, $\operatorname{dim}\left(\operatorname{span}\left\{\bar{B}^{m}, m=0,1\right\}\right)=2$.

We now prove that $\operatorname{dim}\left(\operatorname{span}\left\{\bar{B}^{m}, m=0,1,2,3\right\}\right)=4$. Define:

$$
T=\left(I_{6}+\beta\right) \alpha \text {. }
$$

First, notice that, because $\bar{B}^{0}$ and $\bar{B}^{1}$ are linearly independent and $T$ has rank $4, \bar{B}^{1}$ $\left(=T \bar{B}^{0}\right)$ and $\bar{B}^{2}\left(=T \bar{B}^{1}\right)$ are linearly independent as well. Similarly, because they are rank-4 transformations of linearly independent vectors, $\bar{B}^{2}$ and $\bar{B}^{3}$ will also be linearly independent. But that is not enough. We need to show that $\bar{B}^{3}$ is linearly independent of $\bar{B}^{0}, \bar{B}^{1}$ and $\bar{B}^{2}$, i.e., there do not exist scalars $g_{0}, g_{1}, g_{2}$ and $g_{3}$, such that:

$$
g_{0} \bar{B}^{0}+g_{1} \bar{B}^{1}+g_{2} \bar{B}^{2}+g_{3} \bar{B}^{3}=0 .
$$

Rewrite the latter:

$$
\begin{aligned}
g_{0} & \bar{B}^{0}+g_{1} \bar{B}^{1}+g_{2} \bar{B}^{2}+g_{3} \bar{B}^{3} \\
& =g_{0} \bar{B}^{0}+g_{1} T \bar{B}^{0}+g_{2} T^{2} \bar{B}^{0}+g_{3} T^{3} \bar{B}^{0} \\
& =g_{0} \overline{1}+g_{1} A_{1}+g_{2} A_{2}+g_{3} A_{3},
\end{aligned}
$$

where $\overline{1}$ denotes the unit vector, and $A_{1}, A_{2}$ and $A_{3}$ are vectors obtained by summing the columns of $T, T^{2}$ and $T^{3}$, respectively. Generically, $\overline{1}, A_{1}, A_{2}$ and $A_{3}$ are linearly independent, and, hence, there do not exist linear combinations which equal zero. 